\title{
Impaired hypocretin/orexin system alters responses to salient stimuli in obese male mice
}

\author{
Ying Tan, ${ }^{1,2}$ Fu Hang, ${ }^{1,3}$ Zhong-Wu Liu, ${ }^{1}$ Milan Stoiljkovic, ${ }^{1}$ Mingxing Wu, ${ }^{1,4}$ Yue Tu, ${ }^{1,5}$ Wenfei Han, ${ }^{6}$ Angela M. Lee, ${ }^{7}$ Craig Kelley, ${ }^{8}$ \\ Mihály Hajós, ${ }^{1}$ Lingeng Lu, ${ }^{9}$ Luis de Lecea, ${ }^{10}$ Ivan De Araujo, ${ }^{6}$ Marina R. Picciotto, ${ }^{7}$ Tamas L. Horvath, ${ }^{1}$ and Xiao-Bing Gao \\ 'Department of Comparative Medicine, Yale University School of Medicine, New Haven, Connecticut, USA. ²Department of Neurosurgery, Guizhou Provincial People's Hospital, Guiyang, Guizhou, China. \\ ${ }^{3}$ Guangxi Reproductive Medical Research Center, First Affiliated Hospital of Guangxi Medical University, Nanning, Guangxi, China. ${ }^{4}$ Department of Ophthalmology, Second Affiliate Hospital of Chongqing \\ Medical University, Chongqing, China. ${ }^{5}$ Department of Traditional Chinese Medicine Health Preservation, Second Clinic Medical School, Nanjing University of Chinese Medicine, Nanjing, Jiangsu, China. \\ ${ }^{6}$ Department of Neuroscience, Icahn School of Medicine at Mount Sinai, New York, New York, USA. 'Department of Psychiatry, Yale University School of Medicine, New Haven, Connecticut, USA. 8Joint \\ Biomedical Engineering Program, SUNY Downstate and NYU Tandon, Brooklyn, New York, USA. ${ }^{9}$ Department of Chronic Disease Epidemiology, Yale School of Public Health, New Haven, Connecticut, USA. \\ ${ }^{10}$ Department of Psychiatry and Behavioral Sciences, Stanford University School of Medicine, Palo Alto, California, USA.
}

\begin{abstract}
The brain has evolved in an environment where food sources are scarce, and foraging for food is one of the major challenges for survival of the individual and species. Basic and clinical studies show that obesity or overnutrition leads to overwhelming changes in the brain in animals and humans. However, the exact mechanisms underlying the consequences of excessive energy intake are not well understood. Neurons expressing the neuropeptide hypocretin/orexin (Hcrt) in the lateral/ perifonical hypothalamus (LH) are critical for homeostatic regulation, reward seeking, stress response, and cognitive functions. In this study, we examined adaptations in Hcrt cells regulating behavioral responses to salient stimuli in dietinduced obese mice. Our results demonstrated changes in primary cilia, synaptic transmission and plasticity, cellular responses to neurotransmitters necessary for reward seeking, and stress responses in Hcrt neurons from obese mice. Activities of neuronal networks in the LH and hippocampus were impaired as a result of decreased hypocretinergic function. The weakened Hcrt system decreased reward seeking while altering responses to acute stress (stress-coping strategy), which were reversed by selectively activating Hcrt cells with chemogenetics. Taken together, our data suggest that a deficiency in Hcrt signaling may be a common cause of behavioral changes (such as lowered arousal, weakened reward seeking, and altered stress response) in obese animals.
\end{abstract}

\section{Introduction}

The maintenance of energy balance is critical to survival. The brain has evolved to regulate energy intake and expenditure in vertebrate animals, supporting complex behaviors for seeking, securing, consuming, and storing nutrients efficiently. Throughout natural history, foraging for food in an environment where it is scarce has been one of the major evolutionary forces shaping the function of the brain to promote energy intake and positive energy balance. Therefore, overnutrition, and the epidemic of obesity in humans resulting from access to excessive food, has led to enormous and unprecedented changes in brain functions and behaviors. Both basic and clinical studies show that overnutrition decreases reward seeking $(1,2)$, induces anxiety,

\section{Related Commentary: p. 4540}

Authorship note: $\mathrm{Y}$ Tan and $\mathrm{FH}$ are co-first authors and contributed equally to this work.

Conflict of interest: The authors have declared that no conflict of interest exists. Copyright: ( 2020, American Society for Clinical Investigation.

Submitted: June 19, 2019; Accepted: June 3, 2020; Published: August 17, 2020

Reference information: J Clin Invest. 2020;130(9):4985-4998.

https://doi.org/10.1172/JCl130889. anhedonia, and depression-like behaviors $(3,4)$, attenuates wakefulness during the active phase and increases sleep $(5,6)$, and appears to impair cognitive function (7-9). However, it is not entirely clear how overnutrition or obesity may remodel brain circuits, resulting in altered neuronal and behavioral responses to physiological and environmental cues. Understanding obesity-induced adaptations or maladaptations in the brain is important because such an understanding would reveal how evolution has shaped brain function and also would be critical for the management and treatment of obesity and obesity-induced impairment of behaviors.

The hypocretin/orexin (Hcrt) system in the lateral/perifonical hypothalamus $(\mathrm{LH})$ is a central hub for the integration of a wide range of inputs from brain areas encoding metabolic, behavioral, and environmental cues. This system, in turn, sends efferent fibers throughout the brain (10-12). Hcrt cells regulate both physiological homeostasis and complex behaviors in animals $(13,14)$. Hcrt levels are closely related to metabolic status, and activation of the Hcrt system is required for optimal performance of behaviors necessary for survival, including reward seeking, stress response, and cognition $(13,14)$. Hunger and food restriction are sufficient to increase the levels of Hcrt mRNA and trigger neural plasticity in the Hcrt system $(11,15-18)$. In contrast, obesity leads to downregulation of this neuropeptide in rats and mice in most cases (19-21), with an 
A
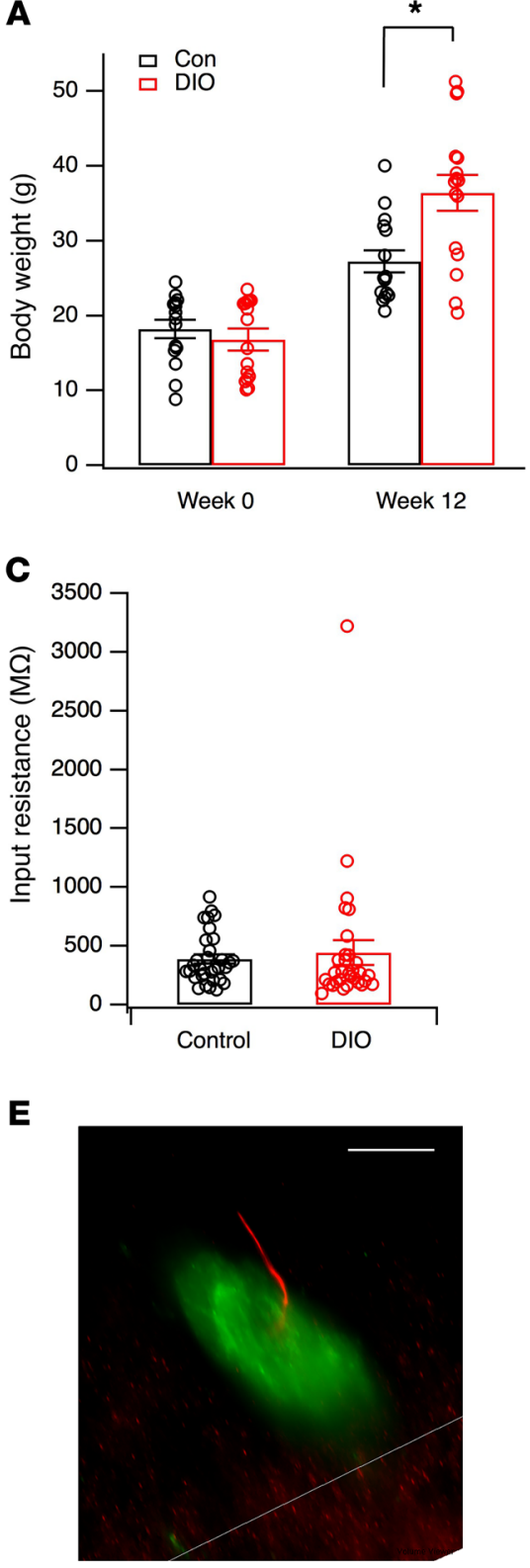

B

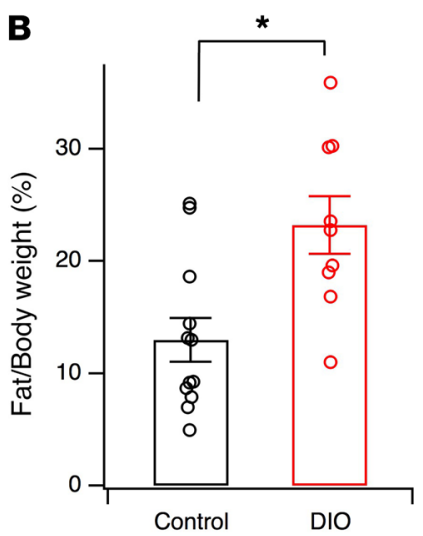

D
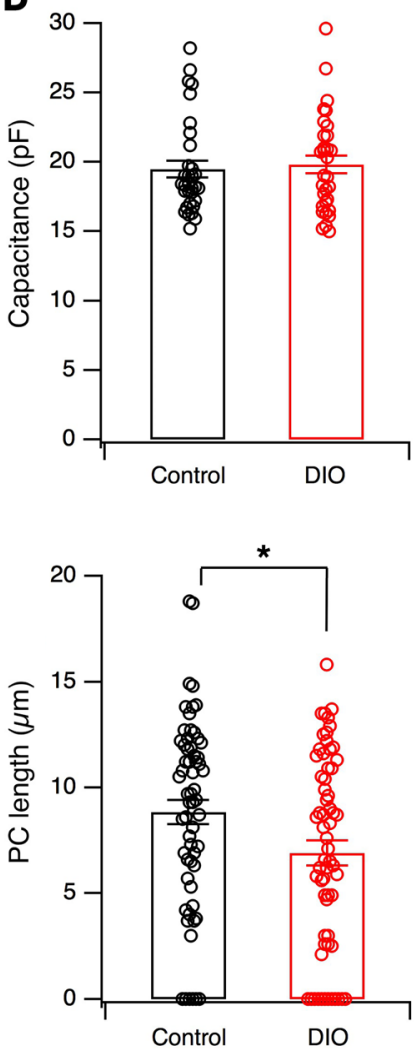

Figure 1. Measurements of body weight, body composition, and basic electrophysiological and morphological parameters in Hcrt cells in control and DIO mice. (A and B) Body weight (control, $n=15$ mice; DIO, $n=16$ mice) and percentage of fat mass (control, $n=12$ mice; DIO, $n=9$ mice) in control (Con) and DIO mice sampled in animals used in our experiments. ${ }^{*} P$ $<0.01$, 2-tailed $t$ test. Data shown in these panels were from different cohorts of mice. (C and $\mathbf{D}$ ) Input resistance and membrane capacitance of Hcrt cells tested with potassium-based pipette solution in control ( $n=32$ cells from 12 mice) and DIO mice ( $n=31$ cells from 12 mice). (E) Lengths of primary cilia (PC) in Hcrt cells. Left: microscopic image of a primary cilium (red) in a Hcrt (green) cell in control Hcrt-GFP mice. GFP was expressed in Hcrt cells under the control of a Hcrt promoter in Hcrt-GFP mice $(39,44,69)$. PC was detected by immunostaining for AC3 using an anti-AC3 primary antibody. Scale bar: $10 \mu \mathrm{m}$. Right: mean lengths of primary cilia in Hcrt cells in control ( $n=61$ cells from 3 mice) and DIO ( $n=63$ cells from 3 mice) mice. ${ }^{*} P<0.05$, 2-tailed $t$ test.

coping in animals. Our results suggest that DIO damages cellular functions of Hcrt neurons, which weakens network activity in the $\mathrm{LH}$ area and hippocampus, thus impairing responses to cocaine and stress in obese mice.

\section{Results}

Since we predicted that obesity weakens the Hcrt system, we evaluated the function of this system in male C57BL/6J mice fed a normal diet or high-fat diet (HFD) for 10 to 12 weeks. In all the experiments reported in this study, mice that gained nearly or more than $100 \%$ of their original body weight following consumption of HFD for 10 to 12 weeks were included in the "obese" group, while those on normal chow were used as controls. In typical cohorts of mice included in our studies, the control group exhibited a lower body weight (control: $27.2 \pm 1.5 \mathrm{~g}, n=15$ mice; DIO: $36.4 \pm 2.4, n=16$ mice, $t=3.244, d f=29, P<0.01$, 2-tailed $t$ test, Figure 1A) and percentage of fat mass than DIO mice (control: $13.0 \% \pm 1.9 \%, n=12$ mice; DIO: $23.2 \% \pm 2.6 \%, n=$ 9 mice, $t=3.245, d f=19, P<0.01$, 2-tailed $t$ test, Figure 1B).

Obesity-induced impairments in cellular functions of Hcrt cells in mice. We started by determining whether DIO induces any changes in electrophysiological and morpho-

exception found in one report (22). In addition to its role in the regulation of energy balance, the Hcrt system is a potent arousal promoter $(13,14)$. Deficiency in Hcrt or its receptor (Hcrtr2 or OXR2) leads to narcolepsy in dogs, mice, and human patients (23-26). Levels of this neuropeptide in the cerebrospinal fluid and activity of Hcrt cells both increase during the active phase (or in response to sleep deprivation) and decrease during the inactive period or sleep (27-30). Selective optogenetic stimulation of Hcrt neurons increases the probability of transition to wakefulness from either slow-wave (SW) sleep or rapid-eye movement (REM) sleep (31). Therefore, it is reasonable to ask whether obesity or overnutrition may induce fundamental changes in the Hcrt system that in turn contribute to alterations of behaviors important for survival.

In this study, we examined the hypothesis that diet-induced obesity (DIO) weakens the Hcrt system and impairs brain responses to salient stimuli that normally lead to reward seeking and stress logical parameters of cell bodies of the Hcrt cells where synaptic and nonsynaptic inputs are integrated and outputs of the neurons are determined. Our data indicated that the input resistance $\left(R_{i n}\right)$ (control, 385.3 $\pm 38.0 \mathrm{M} \Omega, n=32$ cells; DIO, $440.7 \pm 103.7 \mathrm{M} \Omega, n=$ 31 cells, $t=0.5069, d f=61, P=0.61$, 2-tailed $t$ test) and membrane capacitance (control, $19.47 \pm 0.60 \mathrm{pF}, n=32$; DIO, $19.81 \pm 0.64 \mathrm{pF}$, $n=31$ cells; $t=0.3824, d f=61, P=0.70,2$-tailed $t$ test) were not significantly different in Hcrt cells when comparing control and DIO animals (Figure 1, C and D). A growing body of evidence shows that primary cilia contribute to many cellular functions in neurons and are important organelles that are altered as a result of physiological or behavioral challenges (32-36). Among many changes in neuronal morphology induced by the development of obesity in animals, modifications of primary cilia in nerve cells in brain areas regulating energy balance are an emerging hallmark in obese animals. Accordingly, primary cilia are shortened or diminished in 

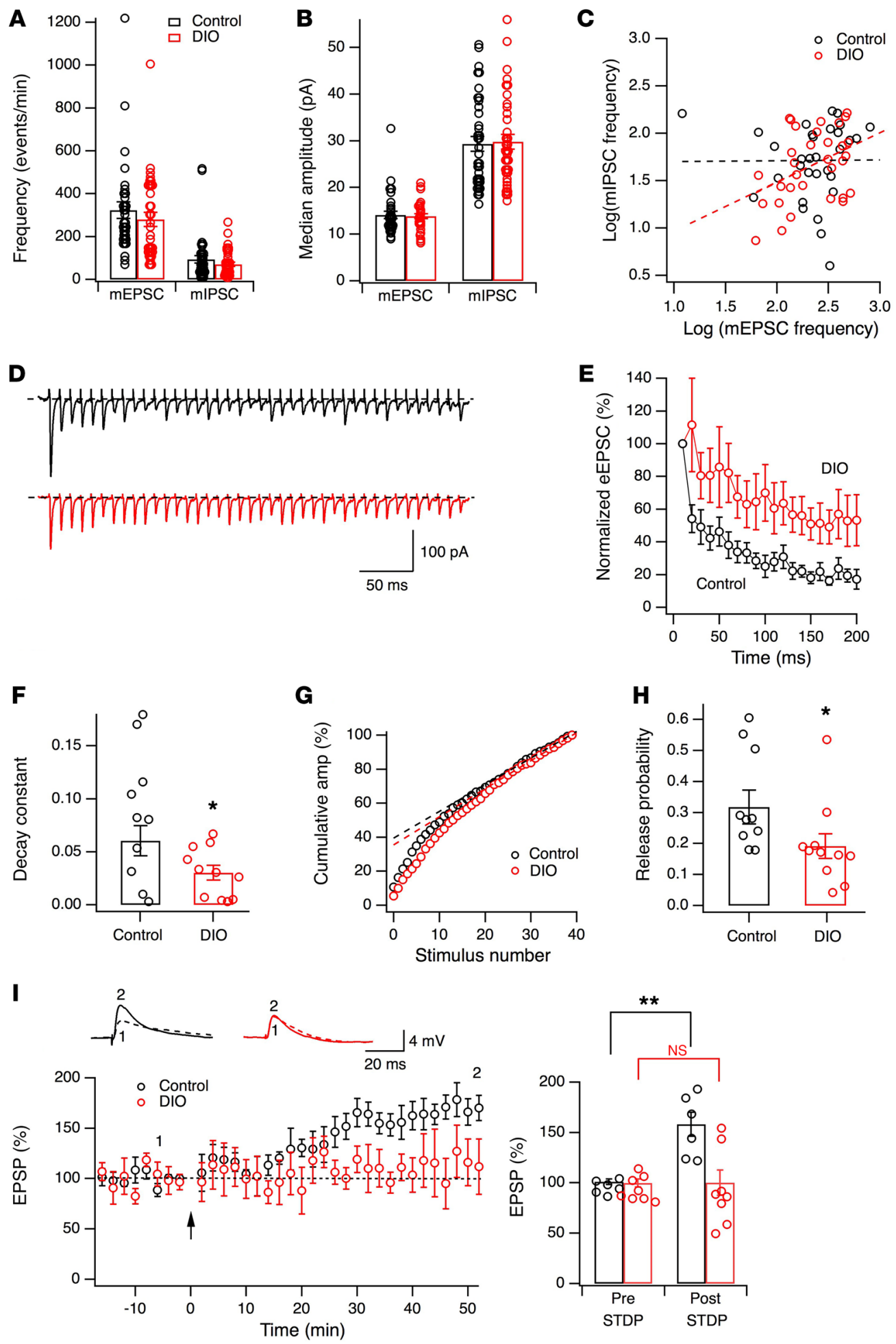

brain areas involved in energy metabolism in obese animal models (32-34). Type 3 adenylyl cyclase (AC3) is predominantly expressed in neuronal primary cilia and is a biological marker of this structure $(32,35,37)$. We therefore used immunocytochemistry for AC3 as a marker for primary cilia in Hcrt cells of Hcrt-GFP mice to measure the length of primary cilia in control versus obese mice as in previous studies (refs. 32, 35 and Figure 1E). The mean length of primary cilia in Hcrt cells was significantly shorter in obese mice compared with control animals (Figure 1E; control: $8.85 \pm 0.58 \mu \mathrm{m}, n=61$ cells from 3 mice; obese mice: $6.91 \pm 0.58 \mu \mathrm{m}, n=63$ cells from 3 mice; $t$ $=2.354, d f=122, P<0.05$, 2-tailed $t$ test).

Next, we determined whether the function of the Hcrt system is altered by the consumption of HFD in these obese mice. Since synaptic inputs onto Hcrt cells contribute to their excitation $(38,39)$, we examined synaptic parameters of glutamatergic and GABAergic synapses onto Hcrt cells (Figure 2, A-C). The frequencies of miniature 

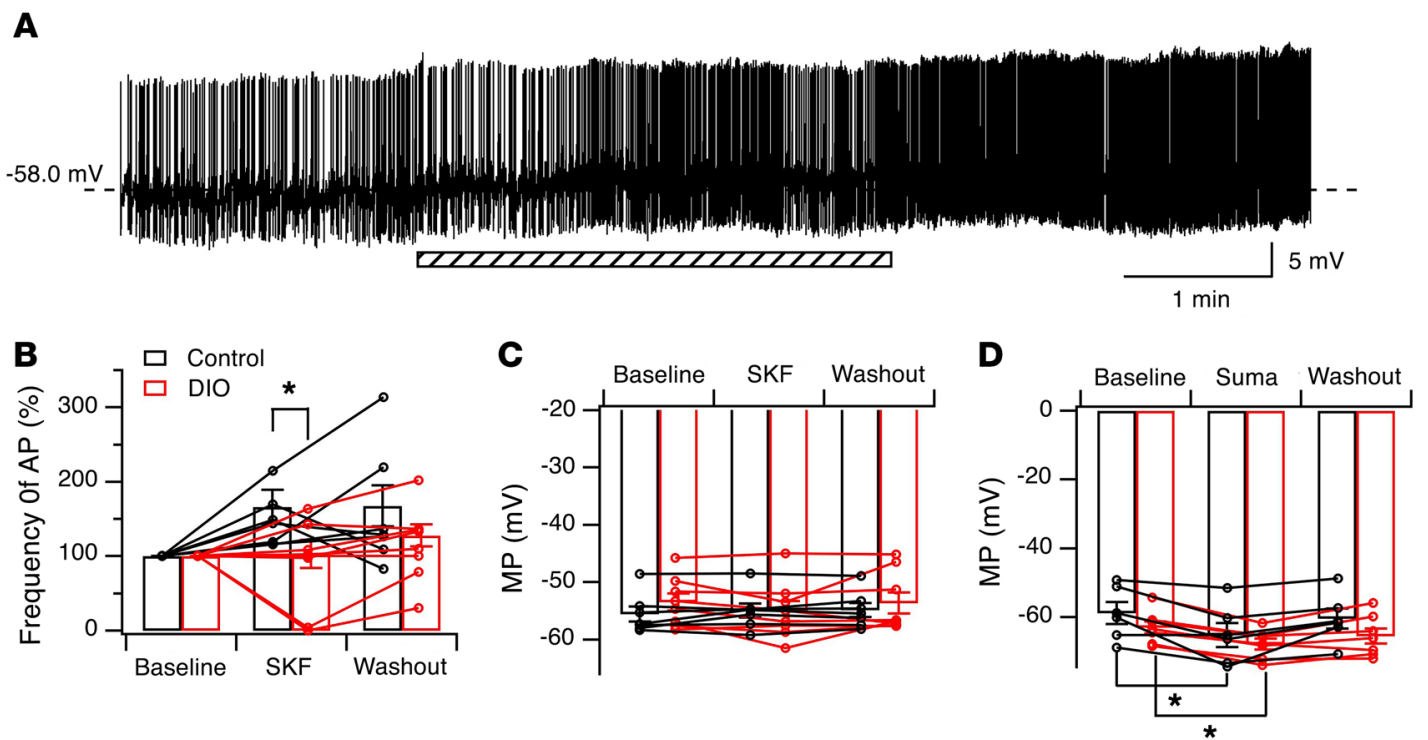

Figure 3. The D1DR- but not the D2DR-mediated effect was impaired in Hcrt cells in obese mice compared with control counterparts. (A) Raw traces of membrane potential (MP) and APs recorded before, during, and after the application of a specific D1 receptor agonist, SKF, in Hcrt cells in control mice. Please note that AP amplitudes were small due to the use of whole-cell recording with a high series resistance to preserve intracellular contents and prevent rundown of AP firing in Hcrt cells in this experiment. The shaded bar indicates the application of SKF. (B and C) Averaged changes in AP frequencies and MP values before, during, and after the application of SKF in Hcrt neurons in control ( $n=7$ cells from 5 mice) and obese (DIO, $n=8$ cells from 6 mice) groups. ${ }^{*} P<0.05$, 2-tailed $t$ test. (D) Averaged MP before, during, and after the application of a selective D2 receptor agonist (Suma) in Hcrt neurons in control and obese mice. ${ }^{*} P<0.01$, repeated-measures 1-way ANOVA.

excitatory postsynaptic currents (control, $322.3 \pm 38.6$ events/min, $n$ $=33$; obese, $279.8 \pm 33.6$ events $/ \mathrm{min}, n=34, t=0.831, P=0.41$, 2 -tailed $t$ test) and miniature inhibitory postsynaptic currents (control, $93.9 \pm$ 17.9 events $/ \mathrm{min}, n=38$; obese, $69.8 \pm 9.8$ events $/ \mathrm{min}, n=38, t=1.178$, $P=0.24$, 2-tailed $t$ test) were not significantly different between control and obese mice (Figure 2A). The averaged medians of miniature excitatory postsynaptic current (mEPSC) (control, $14.1 \pm 0.8 \mathrm{pA}, n$ $=33$; obese, $13.8 \pm 0.5 \mathrm{pA}, n=34, t=0.271, P=0.78$, 2-tailed $t$ test) and miniature inhibitory postsynaptic current (mIPSC) amplitudes (control, $29.3 \pm 1.6 \mathrm{pA}, n=38$; obese, $29.8 \pm 1.6 \mathrm{pA}, n=38, t=0.184$, $P=0.85$, 2-tailed $t$ test) were also not significantly different between control and obese mice (Figure 2B). Next, we examined the balance between excitatory and inhibitory inputs onto Hcrt cells by plotting the logarithmic scales of frequencies of mEPSCs ( $x$ axis) and mIPSCs ( $y$ axis) measured in the same cells from both groups (Figure $2 \mathrm{C}$ ). Correlation analysis with the trend line along the data points from each group (control: $r^{2}=0.0081, F[1,30]=0.00195, P=0.965$; DIO: $r^{2}=$ $0.162, F[1,30]=5.80, P<0.05)$ indicated a significant correlation in obese mice, but not in control, and these 2 groups showed a modest difference between the distributions, with a shift toward the $y$ axis in obese animals $(F[1,60]=3.026, P=0.087$; Figure $2 \mathrm{C})$. Hcrt neurons in obese animals receive fewer inputs overall and also proportionally more inhibitory, and fewer excitatory, inputs than Hcrt cells from control mice. These results suggest that obesity might induce a change in the excitation/inhibition balance among inputs onto Hcrt cells in obese animals, so we examined this hypothesis in more detail.

It has been reported that the releasable pools of neurotransmitters measured with action potential-independent (AP-independent) miniature events might be different from those measured with AP-dependent evoked release (40). Since our data suggest modest changes in the presynaptic parameters of glutamatergic synapses onto Hcrt cells from obese mice, we examined the probability of release from glutamatergic synapses onto Hcrt cells in LH slices from control and obese mice with electric stimulation, using established methods (41). In the presence of picrotoxin $(50 \mu \mathrm{M})$, evoked EPSCs were triggered by a train of high-frequency stimulation (HFS) $(100 \mathrm{~Hz}, 50$ pulses) in Hcrt cells from control and obese mice. The amplitude of evoked EPSCs recorded during the HFS decayed more rapidly in Hcrt cells from control mice than in those from obese animals (control: decay constant $=0.083 \pm 0.019, n=$ 10 from 5 mice; obese: decay constant $=0.030 \pm 0.007, n=11$ from 3 mice; $t=2.657, d f=19, P<0.05$, 2-tailed $t$ test; Figure 2, D-F). This suggests a significant decrease in the probability of release or the size of the readily releasable pool in glutamatergic synapses onto Hcrt cells in obese mice as compared with their controls. To evaluate the amplitude of the responses, we next analyzed the first 40 evoked EPSCs during the HFS in each recorded cell, using established methods modified from published studies (41). The cumulative amplitude of evoked EPSCs recorded from each cell from control and obese mice was measured and normalized (Figure $2 \mathrm{G}$ ). The linear regression of the last 15 points of cumulative eEPSCs was plotted and back extrapolated to the $y$ axis for each cell in each group (ref. 41 and Figure 2G). The $y$ intercept corresponded to the effective readily releasable pool (eRRP or RRP train, hereafter eRRP) for that cell, and the release probability was calculated as the quotient of the value of the first EPSC (normalized to the maximum value) divided by eRRP (41) for cells from both control and obese groups (Figure $2 \mathrm{H}$ ). The mean of release probability $\left(P_{r}\right)$ was significantly lower in obese mice than in controls (control: $P_{r}=0.33 \pm 0.05, n=10$ cells from 5 mice; obese: $P_{r}=0.19$ 
A
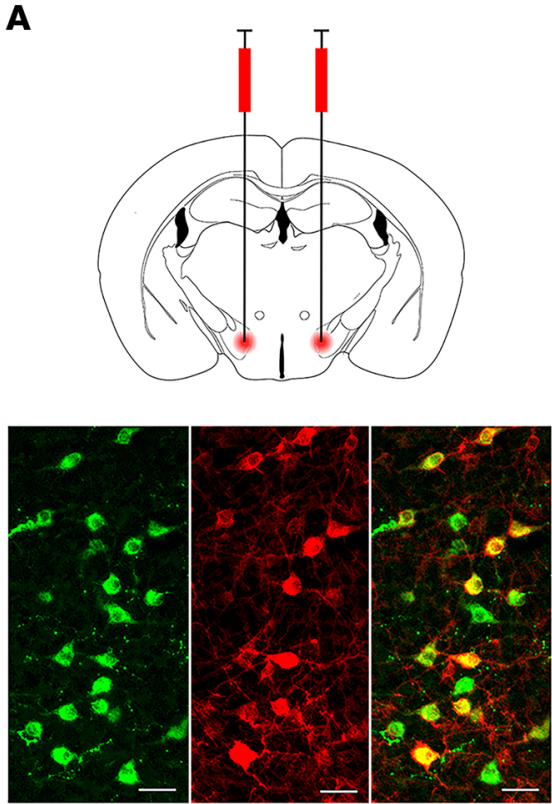

B

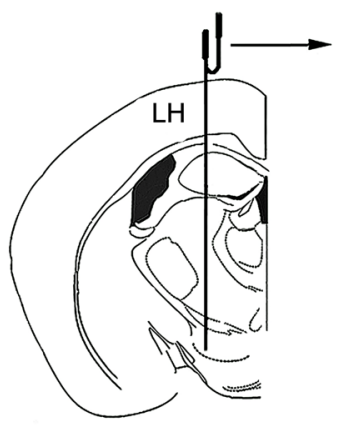

Spontaneous gamma oscillation
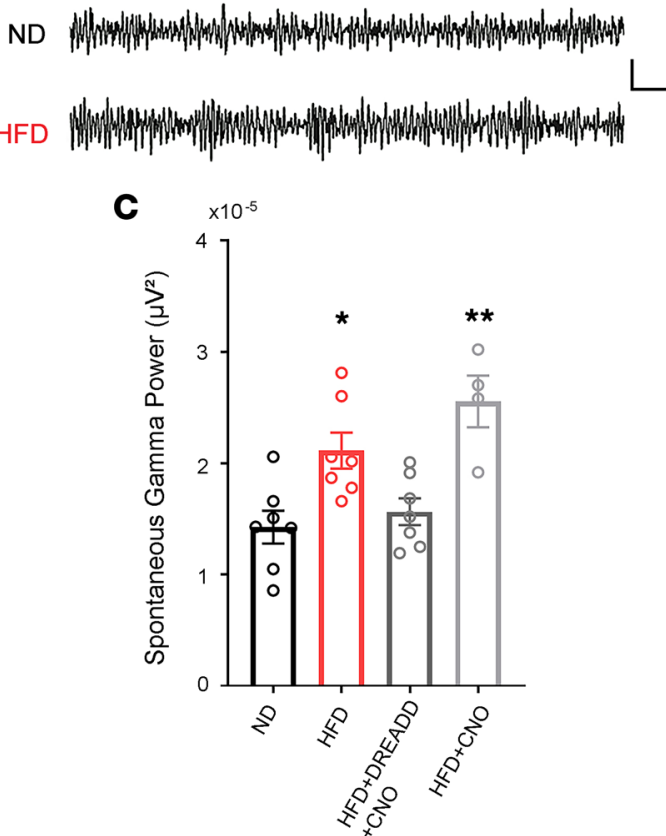

Figure 4. Spontaneous neural oscillations recorded from the LH in mice under urethane anesthesia. (A) Images showing the expression of a stimulatory DREADD receptor (hM3Dq) selectively in Hcrt cells through an AAV viral vector (rAAV5/EF1 $\alpha$-DIO-hM3Dq[Gq]-mCherry) under the control of Cre-recombinase expressed specifically in Hcrt cells in Hcrt-Cre mice. Top: diagram showing the bilateral microinjection of the AAV viral vector into the LH. Bottom: confocal microscopic images of immunostaining of Hcrt (left, green), expression of mCherry (middle, red), and overlap of Hcrt and mCherry signals in Hcrt cells. Scale bars: $40 \mu \mathrm{m}$. (B) Diagram showing the placement of the recording electrode in the LH. Scale bars: $0.05 \mathrm{mV}, 0.1$ seconds. (C) Both obese (HFD, $n=7 \mathrm{mice}$ ) and obese-CNO (HFD+CNO, hM3Dq- mice injected with CNO, $n=4$ mice) animals showed significantly enhanced LH $\gamma$ oscillation power compared with control (ND, $n=7$ mice) and obese mice with a selective activation of Hcrt cells through the hM3Dq receptor (HFD+DREADD+CNO, $n=7$ mice). ${ }^{*} P<0.05$; ${ }^{* *} P<0.01$, 1-way ANOVA. Top: raw traces of typical LFPs in $\gamma$ band from control (ND) and obese (HFD) mice (signal is band-pass filtered between 30 and $90 \mathrm{~Hz}$ ).

$\pm 0.04, n=11$ cells from 3 mice; $t=2.224, d f=19, P<0.05,2$-tailed $t$ test). In addition to changes in basic synaptic properties of glutamatergic synapses, the induction of synaptic plasticity of glutamatergic synapses was examined with a spike-timing dependent (STD) protocol (refs. 42, 43 and Figure 2I). STD plasticity (STDP) was intact in control mice, but not in obese mice (diet and STDP interaction: $F[1,12]=8.307, P<0.05$, 2-way ANOVA; post hoc Holm-Šidák test: control group, before STD versus after STD, $t=$ 3.816, $d f=12, P<0.01$; DIO group, before STD versus after STD $t$ $=0.0042, d f=12, P>0.05)$. Together, our results demonstrate that AP-dependent presynaptic parameters of glutamatergic synapses onto Hcrt cells were significantly altered in obese mice and that the balance between inhibitory and excitatory inputs onto Hcrt cells was modestly shifted toward inhibition. The expression of synaptic plasticity was also impaired in Hcrt cells in obese mice.

The Hcrt system is under the control of many neurotransmitter systems that are involved in homeostatic, behavioral, and environmental responses $(13,14)$. Dopamine (DA) signaling modulates the Hcrt system and is involved in its ability to mediate reward seeking, arousal, and wakefulness $(39,44)$. DAmediated signaling has been shown to regulate behaviors such as reward seeking in a manner responsive to metabolic status (overnutrition or caloric restriction) $(45,46)$. Therefore, we tested the effects of selective D1 and D2 agonists on membrane potential and APs in Hcrt cells from control and obese mice. Consistent with our previous finding that activation of D1 DA receptors (D1DR) mediated the excitation of Hcrt cells by enhancing presynaptic release of glutamate (39), the application of a low dose of the selective D1DR agonist SKF38393 $(5 \mu \mathrm{M})$ in the presence of picrotoxin $(50 \mu \mathrm{M})$ increased the frequency of APs (baseline $=3.17 \pm 0.56 \mathrm{~Hz}, n=7$ from 5 mice) in Hcrt cells in control mice, which was borderline statistically significant (Figure 3, A and B; during application: $147.03 \% \pm 13.64 \%$ of baseline; 10 minutes after washout: $159.46 \% \pm 30.27 \%$ of baseline; $n=7$ cells from 5 mice; $F[2,12]=3.355, P=0.0696$, repeated-measures 1-way ANOVA). In contrast, this dose of SKF38393 $(5 \mu \mathrm{M})$ did not alter AP firing (baseline $=3.11 \pm 0.70 \mathrm{~Hz}, n=8$ from 6 mice) of Hcrt cells from obese mice (Figure 3B; during application: $89.97 \% \pm 20.85 \%$ of baseline; 10 minutes after washout: $115.82 \% \pm 17.69 \%$ of baseline; $n=8$ cells from 6 mice; $F[2,14]=1.226, P=0.3232$, repeated-measures 1-way ANOVA). The percentage changes in AP frequencies induced by SKF in Hcrt cells was significantly different between control and obese mice (control: $147.03 \% \pm 13.64 \%$ of baseline, $n=7$ cells from 5 mice; obese mice: $89.97 \% \pm 20.85 \%$ of baseline, $n=8$ cells from 6 mice; $t=2.217, d f=13, P<0.05$, 2-tailed $t$ test). The membrane potential was not significantly changed by SKF38393 application in Hcrt cells from either group (Figure 3C).

We next tested the effect of the selective D2 DA receptor (D2DR) agonist sumanirole $(2 \mu \mathrm{M})$ on the membrane potential of Hcrt cells in the presence of tetrodotoxin (TTX) (0.5 $\mu \mathrm{M}$; Figure 3D). Consistent with the observation that D2DR activation can alter the membrane potential of Hcrt neurons (47), sumanirole induced a significant hyperpolarization of these cells in both control $(n=6$ cells 
A
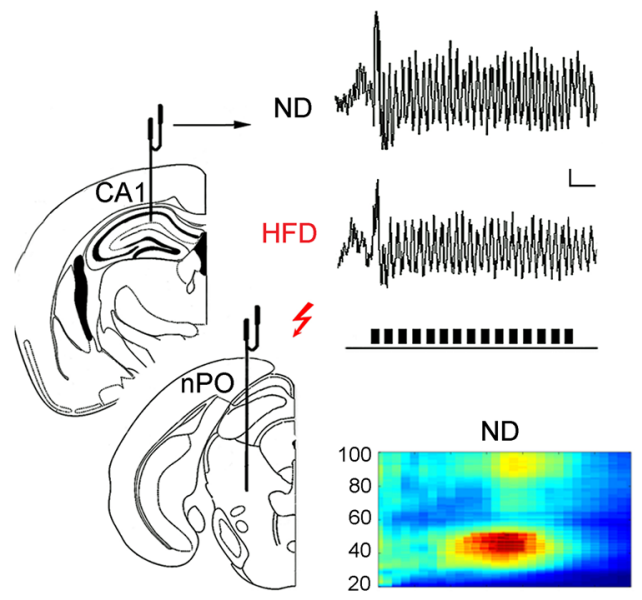

HFD

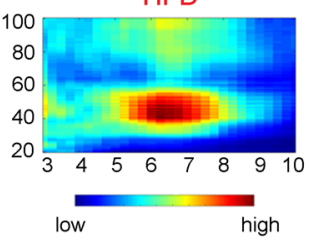

B $\times 10^{-4}$

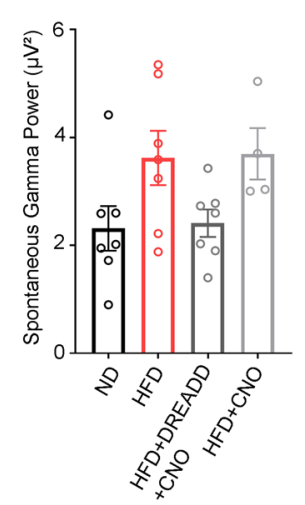

E

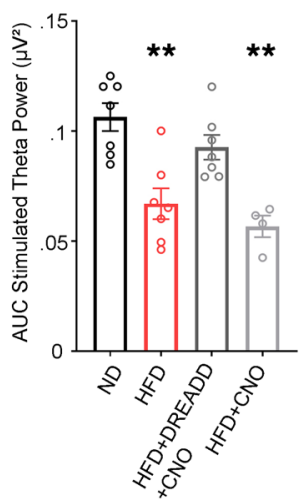

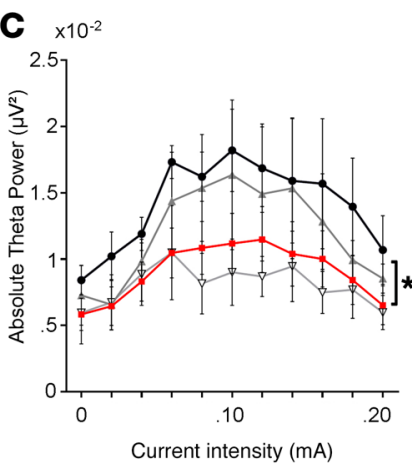

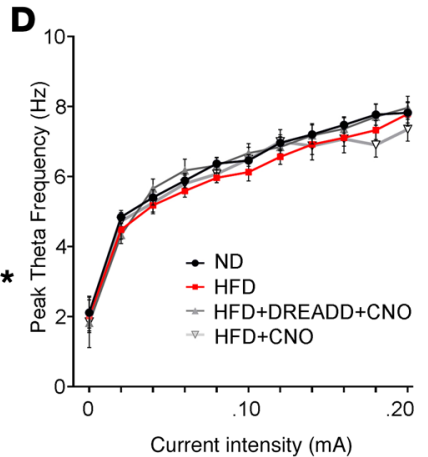

$\mathbf{F}$

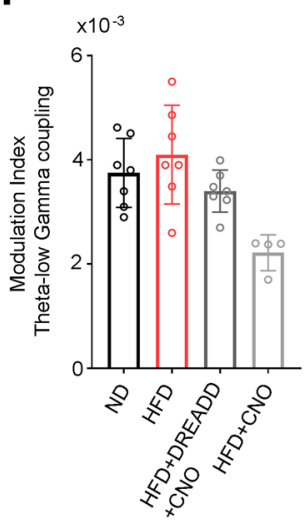

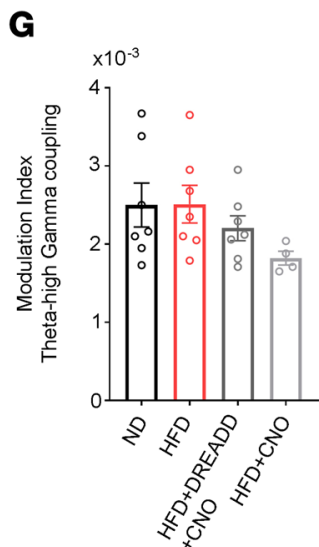

Figure 5. Spontaneous and evoked neural oscillations recorded from the hippocampus in mice under urethane anesthesia. Hippocampal LFPs were recorded from the CA1 region in urethane-anesthetized mice under basal spontaneous conditions and during brainstem nucleus pontis oralis (nPO) electrical stimulation (A, left panel). Typical traces showing elicited $\theta$ oscillation (right panel, upper) and heatmaps (right panel, lower) depicting $\theta$ phase- $\gamma$ amplitude coupling from control (ND, $n=7$ mice) and obese (HFD, $n=7$ mice) mice. Stimulation period with the same current intensity is indicated by train pulses under the lower trace. Scale bar: $0.2 \mathrm{mV}, 1$ second. (B) Spontaneous $\gamma$ oscillation power. (C-E) Stimulus-response relationship plotted for $\theta$ power and peak frequency over increasing stimulus intensities (C and D) and AUC (E) analyses showed a decline in elicited $\theta$ power in obese (HFD, $n=7$ mice) and CNO-treated obese (HFD+CNO, $n=4$ mice) mice (obese hM3Dq-negative mice injected with CNO) compared with controls (ND, $n=7$ mice) and mice with a selective activation of Hcrt cells through the hM3Dq receptor (HFD+DREADD+CNO, $\mathrm{N}=7$ mice). ${ }^{*} P<0.05 ;{ }^{*} P<0.01,1$-way ANOVA. (F and G) The $\theta-$ low $\gamma(\mathbf{F})$ and $\theta-$ high $\gamma$ coupling (G) expressed by the $\mathrm{MI}$ and computed during hippocampal stimulation were not different between the groups (1-way ANOVA test).

from 3 mice; baseline: $-58.9 \pm 3.2 \mathrm{mV}$, sumanirole: $-65.2 \pm 3.5 \mathrm{mV}$, washout: $-60.5 \pm 2.9 \mathrm{mV} ; F[2,10]=7.697, P<0.01$, repeated-measures 1-way ANOVA; Tukey's post hoc test, baseline vs. sumanirole, $q=5.326, d f=10, P<0.01$; baseline vs. washout, $q=1.316, d f=10, P$ $>0.05)$ and obese mice ( $n=7$ cells from 3 mice; baseline: $-63.0 \pm 1.8$ $\mathrm{mV}$, sumanirole: $-67.8 \pm 1.6 \mathrm{mV}$, washout: $-65.5 \pm 2.3 \mathrm{mV} ; F[2,12]=$ $18.55, P<0.01$, repeated-measures 1-way ANOVA; Tukey's post hoc test, baseline vs. sumanirole, $q=8.612, d f=12, P<0.01$; baseline vs. washout, $q=4.446, d f=12, P<0.05)$. The hyperpolarization induced by sumanirole in Hcrt cells was not significantly different between control and obese mice (control: $6.3 \pm 2.1 \mathrm{mV}, n=6$ from 3 mice; obese mice: $4.8 \pm 0.5 \mathrm{mV}, n=7$ from 3 mice; $t=0.7327, d f=11, P=$ $0.479,2$-tailed $t$ test). These results suggest that $\mathrm{D} 1$ receptor-mediated excitation of Hcrt cells was attenuated, while D2-mediated signaling was not altered in obese mice compared with control animals.

Obesity-induced impairments in the activity of neuronal networks in vivo in mice. Since the Hcrt system is weakened in obese mice, it is logical to hypothesize that the target brain regions of this system would be compromised due to impaired output of Hcrt cells. It has been reported that the LH area, in addition to containing the Hcrt cell bodies, has a high level of hypocretin innervation itself (12). Therefore, we determined whether diminished Hcrt neuronal function leads to altered network activity in the hypocretin hub located in the LH area. We performed in vivo recordings of local field potentials (LFPs) in the LH area (Figure 4) in 4 groups of Hcrt-Cre mice under urethane anesthesia: control (ND, saline during recording, $n=7$ mice), obese (HFD, saline during recording, $n=7$ mice), obese-DREADD-CNO (where DREADD indicates designer receptor exclusively activated by a designer drug and $\mathrm{CNO}$ indicates clozapine $\mathrm{N}$-oxide) (HFD+DREADD+ CNO, Hcrt-Cre mice fed on HFD expressing the excitatory DREADD in Hcrt neurons, CNO during recording, $n=7$ mice), and obese-CNO (HFD+CNO, Hcrt-Cre mice fed on HFD without DREADD expression, CNO during recording, $n=$ 4 mice). This design allowed us to determine whether there is a causal relationship between changes in LFPs in the LH area in obese mice and deficiency of the Hcrt system. The expression of a stimulatory DREADD selectively in Hcrt neurons was performed in a subset of obese mice (HFD+DREADD+CNO 

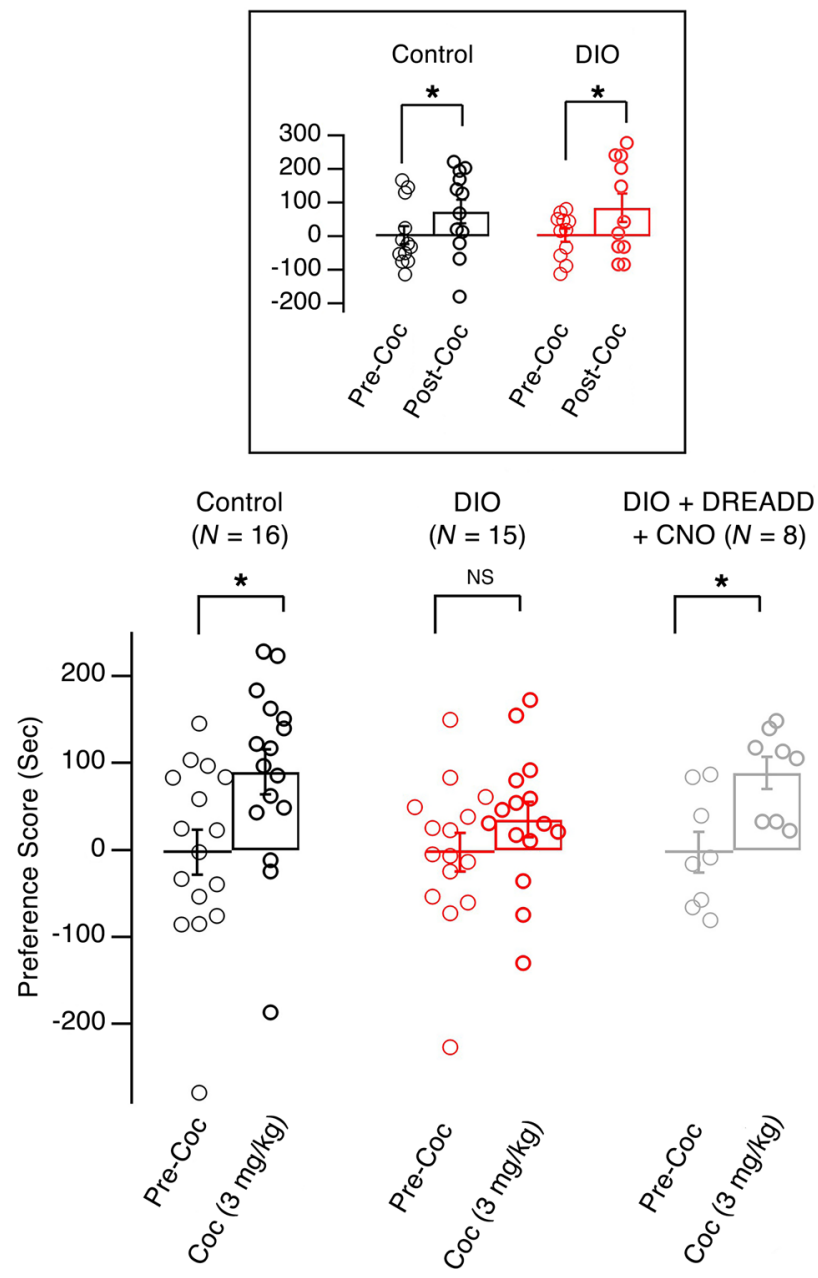

Figure 6. Weakened Hcrt system was responsible for the attenuation of expression of cocaine CPP in obese mice. Bar graph showing the preference scores of 3 groups of mice after the completion of cocaine CPP at a dose of $3 \mathrm{mg} / \mathrm{kg}$ (i.p.): control, obese (DIO), and obese with Hcrt cell activation (DIO+DREADD+CNO). Box, preference scores of control and obese mice after the completion of cocaine CPP at a dose of $10 \mathrm{mg} / \mathrm{kg}$ (i.p.). ${ }^{*} P<0.05$, 2-tailed $t$ test.

group) using a conditional adeno-associated viral (AAV) vector (rAAV5/EF1 $\alpha$-DIO-hM3Dq[Gq]-mCherry) infused into the LH of Hcrt-Cre mice (Figure 4). The designer drug CNO (0.3 $\mathrm{mg} / \mathrm{kg}$, i.p.) or saline was administered to animals 10 minutes after establishing a stable LFP recording, and the effects were measured 20 minutes after the injection of CNO. To exclude a potential nonselective effect induced by the drug, CNO was administered to another subset of obese mice without expression of DREADD (HFD+CNO group). There was a significant difference in spontaneous $\gamma$ oscillation $(30-90 \mathrm{~Hz})$ in the $\mathrm{LH}$ area $(F[3,21]=8.488, P<0.001$, 1-way ANOVA $)$ among the 4 groups of mice (Figure $4 \mathrm{C}$ ). Compared with that in the control groups, $\gamma$ oscillation was significantly increased in obese mice (ND vs. HFD, $q=4.599, d f=21, P<0.05$, Tukey's post hoc test), while selective stimulation of Hcrt cells with CNO in the obeseDREADD-CNO group significantly decreased the $\gamma$ oscillation power to the level of control mice (ND vs. HFD+DREADD+CNO, $q=0.921, d f=21, P=0.914$, Tukey's post hoc test). In contrast, there was no effect of CNO in the "control" obese-CNO group on spontaneous $\gamma$ oscillation, which remained significantly enhanced compared with that of control mice (ND vs. HFD+ CNO, $q=6.436, d f=21, P<0.01$, Tukey's post hoc test). These results suggest that elevated $\gamma$ oscillation in the LH is likely due to impaired Hcrt activity in obese mice.

Next, we determined whether activity of neural circuits outside the hypothalamus is altered as a consequence of impaired output from the Hcrt system in obese mice. The hippocampus is essential for numerous cognitive behaviors. In particular, the dorsal hippocampus is critical for learning the context-drug association measured in drug-seeking behaviors, such as conditioned place preference (CPP) (48-53). Similarly, active coping in the forced swim paradigm also requires intact hippocampal activity (54-57). Importantly, Hcrt neurons provide direct innervation and modulation of the hippocampus (58-61), providing an anatomical rationale for testing the consequences of Hcrt changes on hippocampal activity. We therefore performed LFP recordings in the hippocampal CA1 region and in the LH area in the same mice (Figure 5A).

Spontaneous oscillations in the $\gamma$ band $(30-90 \mathrm{~Hz})$ and high-frequency nucleus pontis oralis (nPO) stimulation-elicited $\theta(3-9 \mathrm{~Hz})$ and $\gamma$ oscillations as well as dynamic interactions between these bands ( $\theta$ phase- $\gamma$ amplitude coupling) were examined in the CA1 region of the hippocampus using methods reported by Stoiljkovic et al. (ref. 62 and Figure 5A). Under basal conditions, spontaneous $\gamma$ power was not significantly different across groups (Figure $5 B ; F[3,21]=1.61 ; P=0.218$, 1 -way ANOVA). Similarly, the elicited $\gamma$ oscillation was not significantly different across groups $(F[3,21]=2.29 ; P=0.107,1$-way ANOVA, data not shown). In contrast, quantitative input-output analysis among the groups showed a significant difference in $\theta$ power $(F[3,198]$ $=12.21 ; P<0.0001,2$-way ANOVA), but not peak frequency $(F[3$, $198]=2.512 ; P=0.098,2$-way ANOVA), in response to varying stimulation intensities (Figure 5, C and D). Post hoc analysis indicated that hippocampal $\theta$ power was significantly lowered in obese and obese-CNO mice when compared with their control groups (ND vs. HFD, $q=6.436, d f=198, P<0.01$; ND vs. HFD+CNO: $q=7.700, d f=198, P<0.01$, Tukey's post hoc test), whereas no significant difference was detected between control and obeseDREADD-CNO mice (ND vs. HFD+DREADD+CNO, $q=2.879$, $d f=198, P=0.18$, Tukey's post hoc test), suggesting that stimulation of Hcrt neurons normalized the difference in hippocampal $\theta$ power observed in obese animals. Accordingly, AUC analysis for elicited $\theta$ (Figure 5E) showed a marked reduction in $\theta$ power in obese and obese-CNO mice when compared with control groups (ND vs. HFD, $q=6.539, d f=21, P<0.01$; ND vs. HFD+CNO, $q=$ 7.037, $d f=21, P<0.01$, Tukey's post hoc test). Again, there was no significant difference between control and obese-DREADDCNO mice (ND vs. HFD+DREADD+CNO, $q=2.283, d f=21, P=$ 0.64, Tukey's post hoc test). There was no effect of CNO alone on LFPs in the control obese-CNO (HFD+CNO) group, ruling out nonspecific effects of $\mathrm{CNO}$ in the current study, even though some recent reports have pointed to possible off-target CNO effects on neural activity $(63,64)$. In addition, the phase-amplitude coupling between hippocampal $\theta$ - and $\gamma$-elicited oscillations was examined (Figure 5, F and G). For $\theta$-low $\gamma$ coupling, it seemed that there was 

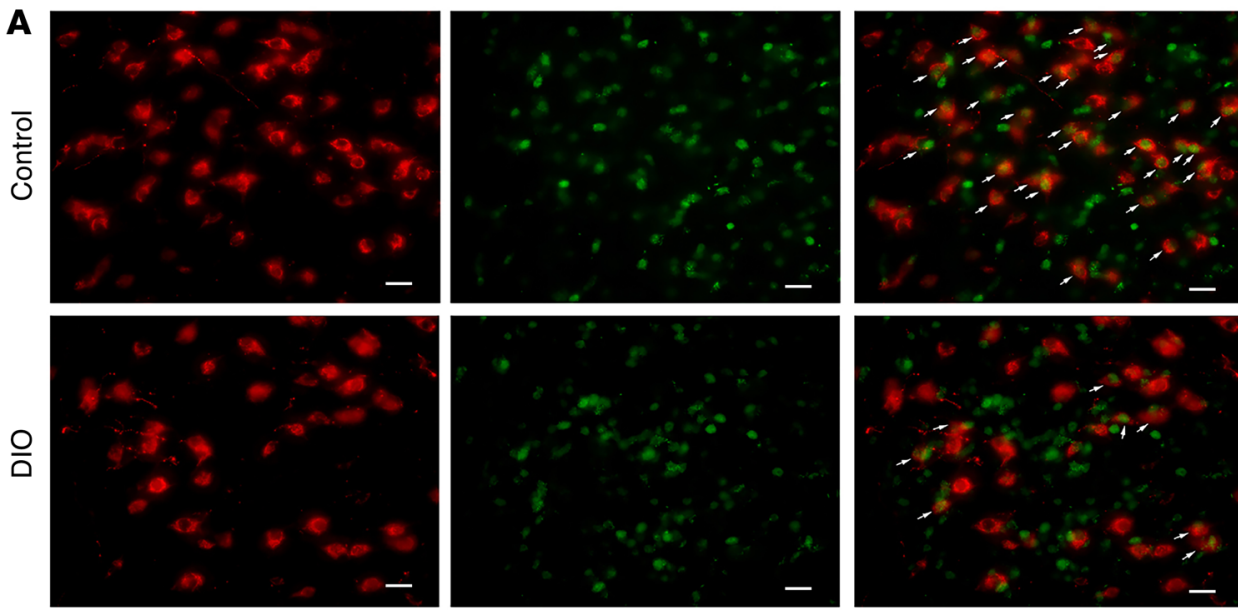

p-CREB

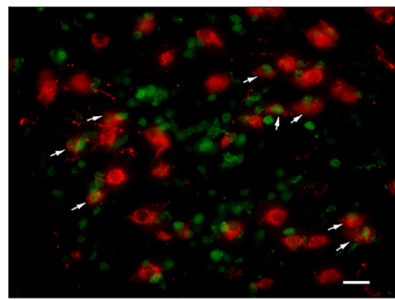

Hort + pCREB

B

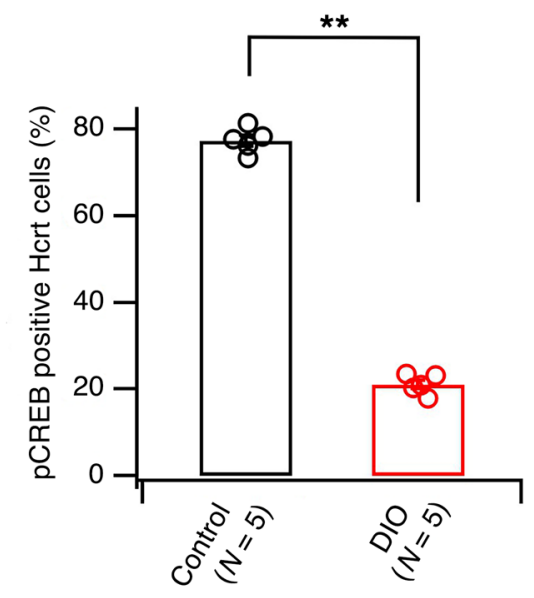

C

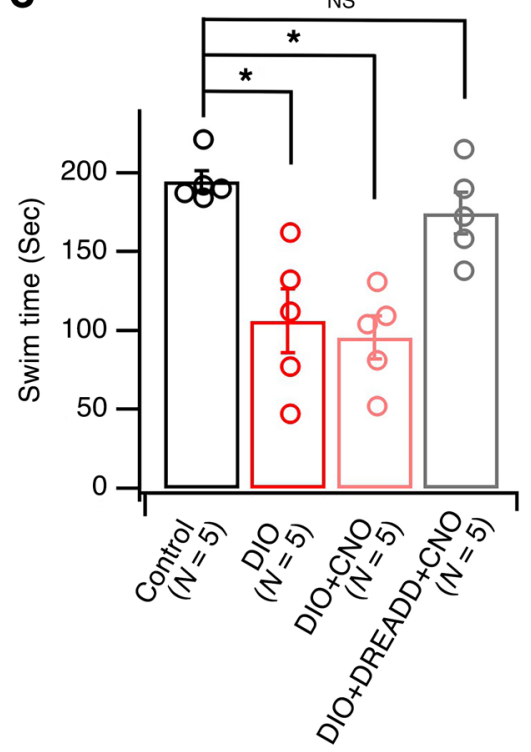

Figure 7. Weakened Hcrt system was responsible for a decreased swim time during forced CWS in obese mice as compared with controls. (A) Images showing immunostaining of the expression of CREB phosphorylation (green) in Hcrt cells (red) in control and obese mice. Arrows indicate positive $\mathrm{p}$-CREB staining (green) in Hcrt-positive (red) cells in control and obese groups. Scale bars: $40 \mu \mathrm{m}$. (B) Bar graph showing percentage of $p$-CREB-positive Hcrt cells in all Hcrt cells 5 minutes after CWS in control and obese (DIO) groups. ${ }^{* *} P<0.01$, 2-tailed $t$ test. (C) Bar graph showing the time spent swimming during a 5-minute session of CWS in 4 groups of mice: control, obese (DIO), obese with a selective activation of $\mathrm{Hcrt}$ cells (DIO+DREADD+CNO), and obese with CNO alone (DIO+CNO). ${ }^{*} P<0.05$, 1-way ANOVA. significant difference among groups $(F[3,21]=6.358, P=0.0031$, 1 -way ANOVA). However, post hoc tests showed differences only in the HFD+CNO group (ND vs. HFD, $q=0.899, d f=21, P=0.379$; $\mathrm{ND}$ vs. HFD+DREADD+CNO, $q=1.575, d f=21, P=0.243$; ND vs. HFD+CNO, $q=3.307, d f=21, P=0.01$, Dunnett's post hoc test), which may be due to a small sample size in this group. For $\theta$-high $\gamma$ coupling, there was no significant difference among groups $(F[3$, 21] $=1.588, P=0.222$, 1-way ANOVA). The overall conclusion is that the phase-amplitude coupling between hippocampal $\theta$ - and $\gamma$-elicited oscillations generally did not differ among groups. Together, these data suggest that weakened Hcrt output in obese animals appears to lead to lowered $\theta$ activity in the hippocampus.

In summary, deficiency in activity of the Hcrt system appears to result in alterations in downstream neuronal networks, both in the LH area and in other brain areas, such as the hippocampus.

Obesity-induced impairments in behaviors mediated by the Hort system in mice. The Hcrt system contributes to processing of natural rewards and the development of drug addiction (65-67); it also undergoes synaptic plasticity following cocaine CPP or selfadministration $(68,69)$. Cocaine-seeking behaviors such as CPP can be used as a bioassay to determine whether circuits encoding rewards are functioning normally.

At a suprathreshold dose $(10 \mathrm{mg} / \mathrm{kg}$, i.p.), cocaine induced CPP in both control and obese mice (Figure 6, box). However, at a lower dose (3 mg/kg, i.p.), cocaine CPP was intact in control mice (Figure 6; precocaine preference score: $-2.47 \pm 26.11 \mathrm{sec}-$ onds; postcocaine preference score: $89.8 \pm 26.11$ seconds; $n=16$ mice; $t=2.798, d f=15, P<0.05$, paired $t$ test), but not in obese animals (Figure 6; precocaine preference score: $-2.46 \pm 22.04$ seconds; postcocaine preference score: $34.98 \pm 20.10$ seconds; $n$ $=15$ mice; $t=1.469, d f=14, P=0.164$, paired $t$ test). In a parallel set of experiments, we measured induction of c-Fos expression in Hcrt cells following acute exposure to the low dose of cocaine (3 $\mathrm{mg} / \mathrm{kg}$, i.p.), which indicated decreased activation of Hcrt cells in obese mice in response to cocaine exposure compared with controls (Supplemental Figure 1; supplemental material available online with this article; https://doi.org/10.1172/JCI130889DS1). To determine whether the decreased CPP at a threshold dose of cocaine is due to changes in function of Hcrt cells, we expressed a stimulatory DREADD receptor selectively in these neurons 
Behavioral changes in animals

(reward seeking/motivation, stress responses, learning deficiency)

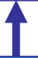

Diminished generalized arousal levels

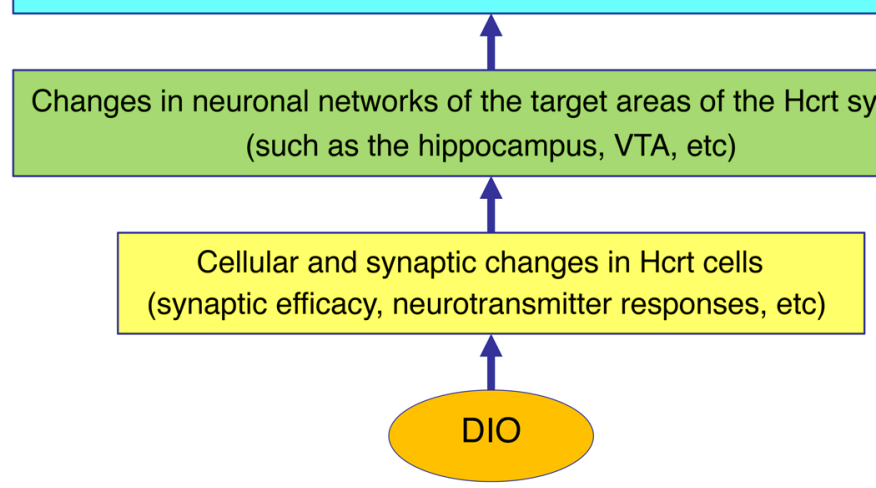

Figure 8. Paradigm depicting the potential consequences of changes that occur in Hcrt cells in obese mice. These changes can lead to altered activities in neuronal networks downstream of the Hcrt system, which would affect arousal levels as well as animal behaviors governed by the Hcrt system. in Hcrt cells by $77.37 \% \pm 1.31 \%(n=5$ mice $)$, while CWS only increased p-CREB levels by $21.07 \% \pm 1.02 \%$ ( $n=5$ mice) in obese mice (Figure 7, $\mathrm{A}$ and $\mathrm{B} ; t=34.02, d f=8, P<0.0001,2$ tailed $t$ test). The expression of $\mathrm{c}$-Fos induced by CWS in Hcrt cells was also decreased in DIO mice as compared with controls (Supplemental Figure 4). Consistent with decreased activation of Hcrt cells in obese mice, swim time, which is thought to represent an active coping strategy (72), was significantly decreased in obese mice (106.0 \pm 20.2 seconds; $n=5$ mice) as compared with control mice fed the normal diet (194.8 \pm 6.7 seconds; $n=5$ mice). CNO administration to obese Hcrt-Cre, but not Cre-negative, mice infused with a Gq-coupled DREADD reversed the decrease in swim time (Figure 7C; DIO+DREADD+CNO mice: $174.6 \pm 13.2 \mathrm{sec}-$ onds, $n=5$; Cre-negative DIO+CNO mice: 95.4 \pm 13.4 seconds, $n=5$ mice $)(F[3,16]=12.06, P<$ 0.01, ANOVA; post hoc Dunnett's test: control vs. DIO, $q=4.416, d f=16, P<0.01$; control vs. $\mathrm{DIO}+\mathrm{CNO}, q=4.943, d f=16, P<0.001$; control vs. DIO+DREADD+CNO, $q=1.005, d f=$ using a conditional AAV vector (rAAV5/EF1 $\alpha$-DIO-hM3D$\mathrm{q}[\mathrm{Gq}]$-mCherry) infused into the LH of Hcrt-Cre mice (Figure 4). CNO (0.3 mg/kg, i.p.) was applied 15 minutes before each CPP training session with a low dose of cocaine $(3 \mathrm{mg} / \mathrm{kg})$. Significant cocaine CPP was observed in obese mice expressing the activating DREADD and treated with CNO when the low dose of cocaine was used (Figure 6; precocaine preference score: $-2.57 \pm$ 23.40 seconds; postcocaine preference score: $88.80 \pm 18.21 \mathrm{sec}-$ onds; $n=8$ mice; $t=6.836, d f=7, P<0.05$, paired $t$ test). These data suggest that the weakened Hcrt signaling may be responsible for attenuated cocaine CPP in obese mice, indicating decreased sensitivity to reward in these animals.

In addition to deficits in reward seeking, obese mice exhibit altered responses to stress, another important set of behaviors dependent on function of the Hcrt system $(3,4,66)$. To determine whether alterations in Hcrt function are responsible for altered behavioral responses to stress in obese mice, the total time spent swimming (swim time) in the forced cold-water swim (CWS) test $(70,71)$ was evaluated in male control and obese mice. The time spent swimming is suggested to be indicative of an active coping strategy in response to this stressor $(72,73)$. To determine whether Hcrt neurons are recruited during exposure to an inescapable stressor, we measured phosphorylation of the transcription factor CREB ( $\mathrm{p}$-CREB) on serine 133, a rapidly induced biochemical marker of neuronal activity. p-CREB is activated by stress exposure and drug addiction and is critical for the induction of synaptic plasticity, as previously reported by others and us $(69,74,75)$. A 5-minute CWS rapidly induced a significant increase in p-CREB in Hcrt cells, as demonstrated with dual immunocytochemistry using antibodies against Hcrt and p-CREB (Supplemental Figure 2). p-CREB induction peaked at 5 minutes after exposure to CWS and decayed by 15 and 30 minutes after CWS exposure (Supplemental Figure 3). In control mice, CWS increased p-CREB levels
$16, P=0.632)$. CNO at the dose used in this study did not have a significant effect on swim time, as reported previously $(63,64)$. These results suggest that the response to an acute stressor was altered in obese mice, due to decreased activity of Hcrt neurons and diminished output of the hypocretin system.

\section{Discussion}

In this study, we demonstrate that the Hcrt system is compromised in obese mice and that deficiency in the Hcrt system may be responsible for altered behaviors in obese animals (Figure 8). Morphologically, primary cilia were shorter in Hcrt cells from obese mice, suggesting that these cells might be compromised in the ability to sense the local environment in the $\mathrm{LH}$ and might be responsible for impaired neuronal functions in Hcrt cells, as reported previously in other neuronal systems (32-36). Electrophysiologically, glutamatergic inputs onto Hcrt cells were weakened and expression of synaptic potentiation was compromised in obese mice. With respect to neuromodulators that normally regulate the activity of Hcrt neurons, D1DR-mediated excitation, but not DRD2-induced inhibition, was decreased in Hcrt cells from obese animals. These changes lead to decreased activity of the Hcrt system and may be responsible for a marked elevation of spontaneous $\gamma$ oscillation in the LH area and decrease in evoked $\theta$ oscillation in the hippocampus. Taken together, these observations demonstrate enhanced inhibitory tone in the LH of obese animals and lessened output to target brain areas (such as the hippocampus). These deficits at the cellular and circuit levels contribute to a reward deficit and altered response to acute stress in obese mice, as demonstrated by the ability of chemogenetic activation of Hcrt neurons to reverse these obesity-dependent phenotypes.

The shortened primary cilia observed in Hcrt cells from obese mice are consistent with published studies demonstrating shortened primary cilia in hypothalamic neurons regulating energy balance in several animal models of obesity (32-34). Although the 
consequences of this morphological change are not yet entirely clear, primary cilia can regulate several aspects of neuronal function (32-36). Primary cilia promote the integration of adult-born neurons into existing neuronal circuits by extending dendrites and forming new synapses (35). Relevant to energy balance, selective inhibition of AC3 in primary cilia in MC4R-expressing Sim1 neurons in the periventricular nucleus (PVN) led to an enhancement in body weight in animals (34). In the current study, the observation of shortened primary cilia in Hcrt cells from obese animals provides a potential cellular mechanism that may underlie modified synaptic transmission and plasticity in these cells and altered activity in neuronal circuits downstream of the Hcrt system and that may contribute to behavioral changes in obese animals. However, additional studies will be required to establish a causal relationship between this morphological change and observed network-level changes in activity of the Hcrt system.

It has been reported that substantial changes occur in Hcrt mRNA and protein levels in genetic or DIO models (19-21). Hcrt neurons from obese leptin knockout mice (OB/OB) or obese mice on HFD receive an increased density of cannabinoid receptor type 1-expressing (CB1-expressing) inhibitory inputs (76). Consistent with this morphological observation, the frequency of mIPSCs was significantly higher, and CB1 agonists were able to suppress the activity of inhibitory synapses more effectively, in $\mathrm{OB} / \mathrm{OB}$ mice (76). A recent study showed that a 1-week consumption of HFD could induce short-term depression of glutamatergic synapses onto Hcrt cells (77), which was not seen in rats treated with HFD for 4 weeks. In the current study, chronic ( $\sim 3$ months) consumption of HFD impaired signaling of glutamatergic synapses onto Hcrt cells through multiple mechanisms. There was a decrease in the probability of glutamate release from presynaptic terminals, induction of STDP was diminished, and the balance between excitatory and inhibitory inputs onto Hcrt cells was likely shifted toward inhibition. The differences between the current study and those reported in rats on HFD (77) may be due to species differences between mice and rats, but also in part to the time course of HFD consumption (12 weeks in this study compared with a short-term administration in experiments in rats). We also excluded all HFD-resistant mice that did not double their body weight, whereas all rats on HFD were included without consideration of the development of obesity in Linehan et al. (77). Other limitations of the current study include the focus on the Hcrt system in animals that have already developed obesity. It is not clear how the Hcrt system changes during the development of obesity, especially during initial exposure to HFD. HFD is palatable and is rewarding, which might induce additional forms of plasticity in the Hcrt system, as has been reported previously in cocaine-seeking rodents $(68,69,77)$. How the Hcrt system changes during the entire process from excessive consumption of HFD to blunting of reward seeking in obese animals is not yet clear and warrants additional studies. In addition, there are also many other physiological changes in obese animals, including hyperglycemia/diabetes, hypertriglyceridemia, etc. It is possible that these metabolic changes may also have adverse effects on the Hcrt system that have not been examined in this study.

The effects of obesity on the CNS have attracted substantial attention in recent years due to the increasing prevalence of obesity in humans. Originally, studies of neuronal changes induced by diet- and genetically induced obesity focused on brain areas responsible for energy balance, diabetes, and cardiovascular function (78-80). More recently, there has been an increasing focus on diet-induced changes in cognitive functions (7-9). There is evidence that obese animals demonstrate impaired associative learning and memory, altered stress responses, and weakened reward seeking, among other attributes $(1-4,7,8)$. One possibility is that obesity may induce specific effects on each brain area involved in learning and memory, stress response, and reward seeking (1-4, $7,8,81)$. An alternative explanation is that obesity may induce changes in one, or a few, brain areas that project widely in the CNS and can then regulate the functions of many brain areas and related behaviors concurrently. We propose that the Hcrt system may be one of these candidates.

One of the overarching physiological explanations for the broad effects of DIO could be that obesity causes a lowered generalized arousal state in animals, which is responsible for the dysregulated sleep/wake cycle, daytime sleepiness, decreased reward seeking, altered stress response, and cognitive deficits reported in previous studies (1-6). Impairment of the Hcrt system appears to underlie hypoarousal in obese mice, since this can be reversed by chemogenetic stimulation of Hcrt neurons or administration of the psychostimulant modafinil (Supplemental Figure 5). A recent report indicated that the Hcrt system mediates both preference and avoidance behaviors, depending on the inputs onto Hcrt cells (82). The results reported here demonstrate that synaptic inputs onto Hcrt neurons are altered in obese animals. Although the origins of the altered synaptic inputs have not yet been identified, it is very likely that these changes contribute to behavioral phenotypes described in obese animals $(3,4)$.

The results reported here may also help in the understanding of other physiological and behavioral phenotypes of obese animals. The Hcrt system promotes energy intake and expenditure $(13,14)$. A recent study suggests that activation of the Hcrt system can inhibit food intake (83). Thus, a weakened Hcrt system would disinhibit feeding in obese animals. This may contribute to hyperphagia and altered energy metabolism in obese animals and human patients.

In summary, the results reported here indicate that DIO results in substantial impairment in Hcrt neuronal activity, which in turn dysregulates activity locally in the $\mathrm{LH}$ and in brain areas to which these cells project, ultimately causing behavioral changes, including deficiency in reward seeking and altered stress response. These results may provide new strategies for the treatment of obesity and the management of diseases resulting from obesity.

\section{Methods}

Detailed information is provided in the Supplemental Methods.

Animals, stereotaxic viral injections, and behavioral paradigms. C57BL/6J mice (4-6 months old) expressing GFP (Hcrt-GFP) (18, 38, $39,44)$ or Cre-recombinase (Hcrt-Cre) (82) selectively in hypocretin neurons under the control of a specific hypocretin promoter were used in this study. Mice were group housed and maintained on a 12-hour light/12-hour dark cycle (lights on at 6:00 am) with free access to food and water under the care of Yale Animal Resources Center personnel.

At an age of about 21 days, male C57BL/6J mice were randomly divided into 2 groups: control, mice fed with normal chow ad libitum for 10 to 12 weeks; and DIO, mice fed with a HFD with $45 \%$ of 
calories from fat (D12451, Research Diets Inc.) ad libitum for 10 to 12 weeks. Only those mice in the obese group with a body weight gain of more than $100 \%$ were included in this study together with all animals in the control group. At 12 weeks on the diet, control and HFD-treated mice were scanned by nuclear MRI (EchoMRI) in order to determine their whole-body composition.

The surgery for stereotaxic injection of AAV viral vector (rAAV5/ EF1 $\alpha$-DIO-hM3Dq[Gq]-mCherry) was performed as reported by Han et al. (84). The expression of viral vectors was confirmed by dual immunocytochemical examinations of Hcrt and mCherry in injected mice. Cocaine CPP was performed as we reported previously (69). Forced CWS was performed as reported elsewhere previously (70, 71). Five minutes after the test, all animals were scarified under deep anesthesia, followed by transcardial perfusion of fixative containing $4 \%$ paraformaldehyde in $0.1 \mathrm{M}$ sodium phosphate buffer ( $\mathrm{PB})(\mathrm{pH}$ 7.4). Brains were collected for the further immunocytochemical experiments on expression of p-CREB and c-fos as reported previously by us $(39,69)$.

Immunocytochemistry. Brains from all groups of animals collected in our study were cut into sections (50 $\mu \mathrm{m}$ thick) on a vibratome and were washed in a buffer containing $0.1 \%$ lysine, $1 \%$ BSA, $0.1 \%$ Tris, and $0.4 \%$ Triton $X-100$, after which they were blocked with $2 \%$ normal horse serum and were incubated overnight in primary antibodies for orexin-B (Santa Cruz Biotechnology Inc.; catalog sc-8071, goat, 1:2500) plus p-CREB (Ser133) (Cell Signaling Technology; catalog 9191 and 9198, rabbit, 1:100) or AC3 (marker for primary cilium, Abcam; catalog ab125093, rabbit, 1:200). After several washes with $\mathrm{PB}$, sections were incubated in secondary antibodies (1:250) conjugated to Alexa Fluor 488 and Alexa Fluor 594 (Invitrogen) for 2 to 3 hours. Specimens were examined with an FV 300 confocal laser scanning microscope (Olympus America) or the Keyence Microscope System (Keyence Corp.). For cell counting, at least 6 sections from each animal were taken at the level of the LH, and the number of CREB/hypocretin-positive neurons were counted and compared with the total number of hypocretin neurons. For the measurement of primary cilia in Hcrt cells, Z-scanning was performed along the whole depth of the cell and a 3D reconstruction of Hcrt cells with primary cilia was performed with Image (NIH). The lengths of primary cilia were presented as mean \pm SEM for each group, and $t$ test was used to determine significance.

In vitro electrophysiology. Coronal hypothalamic slices, $300 \mu \mathrm{m}$ thick, were cut from mice expressing GFP exclusively in Hcrt neurons, as we reported previously $(39,44,69)$. Whole-cell voltage clamp (at $-60 \mathrm{mV}$ or at $\mathrm{O} \mathrm{mV}$ ) was performed to observe miniature excitatory and inhibitory postsynaptic currents with a MultiClamp 700A amplifier (Axon Instruments, Molecular Devices, LLC), as described previously $(39,44,69)$. To monitor evoked EPSCs, a bipolar tungsten electrode with a small distance of $50 \mu \mathrm{m}$ was placed across an identified dendrite of Hcrt cells about 50 to $100 \mu \mathrm{m}$ away from the soma, where whole-cell recording was performed. After a stable recording of eEPSCs was achieved, the stimulating intensity (20 to $50 \mu \mathrm{A})$ was adjusted to trigger eEPSCs at about $30 \%-50 \%$ of their maximal amplitudes. The stimulation and analysis of release probability were performed as reported by Thanawala and Regehr (41), with modifications. In LTP experiments, a STD protocol was used, as reported elsewhere $(42,43)$. Since AP firing in Hcrt cells could run down rapidly due to the loss of intracellular contents during conventional whole-cell recording with a low series resistance (10-20 M $\Omega$ ), as reported previously by others and us $(85,86)$, we used a modified whole-cell recording configuration with a high series resistance (30-40 M $\Omega$ ) to eliminate the rundown of AP firing in Hcrt cells in this experiment by partially rupturing neuronal membrane after a gigaseal formation. In all experiments, only recordings with stable series resistance were accepted (the change in series resistance was less than 20\%).

All data were sampled at $10 \mathrm{kHz}$ and filtered at $3 \mathrm{kHz}$ with an Apple computer using AxoGraph X (developed by John Clements, AxoGraph, Inc.). Electrophysiological data were analyzed with AxoGraph X and plotted with Igor Pro software (WaveMetrics). Specifically, mEPSC was analyzed with an event-detection package provided in AxoGraph X, as reported previously $(39,44,69)$.

In vivo electrophysiology. Neurophysiological in vivo recordings were carried out in mice anesthetized with urethane $(1.5 \mathrm{~g} / \mathrm{kg}$, i.p.) (62). After achieving a stable plane of anesthesia, mice were placed in a Kopf stereotaxic frame on a temperature-regulated heating pad (Physitemp Instruments Inc.) set to maintain body temperature at $37-38^{\circ} \mathrm{C}$, and unilateral craniotomies were performed above the hypothalamus, hippocampus, and rostral pons. After surgery, 2 bipolar concentric electrodes (NE-100X, Rhodes Medical Instruments) were inserted into lateral hypothalamic $(1.5 \mathrm{~mm}$ posterior, $1.0 \mathrm{~mm}$ lateral and $4.7 \mathrm{~mm}$ dorsoventral) and hippocampal CA1 (2.0 $\mathrm{mm}$ posterior, $1.5 \mathrm{~mm}$ lateral and $1.5 \mathrm{~mm}$ dorsoventral) regions. Coordinates for each target area were taken from Paxinos and Franklin's mouse brain atlas (87) and referenced relative to bregma and brain surface. Animals were allowed to stabilize before recording was begun. Ten minutes after establishing the stable LFPs, they were i.p. injected with saline (ND and HFD mice) or $0.3 \mathrm{mg} /$ $\mathrm{kg}$ CNO (HFD-DREADD and HFD-CNO mice). The recording of spontaneous LFPs in the LH and CA1 started 20 minutes following injections of saline or CNO in each mouse and lasted for 15 minutes before initiation of elicited hippocampal oscillations by electrical stimulation of nPO, as described previously (62). Briefly, the hypothalamic electrode was repositioned into the nPO $(4.0 \mathrm{~mm}$ posterior, $1.2 \mathrm{~mm}$ lateral, and $3.3 \mathrm{~mm}$ dorsoventral), and stimulation consisted of a train of $0.3 \mathrm{~ms}$ square pulses delivered over a period of 6 seconds at a rate of $250 \mathrm{~Hz}$ repeated every 100 seconds using an Isoflex stimulus isolator unit (A.M.P.I. Instruments). The stimulating current was increased stepwise from 0.0 to $0.2 \mathrm{~mA}$ in 0.02 $\mathrm{mA}$ increments and repeated in 3 cycles in order to establish a stimulus-response relationship for both total power and peak frequency in the $\theta$ band. The protocol for measuring evoked LFPs lasted for about 50 minutes. All recordings of spontaneous and evoked LFPs were performed within the effective time frame of $\mathrm{CNO}$ reported in mice (88-91). Throughout the duration of the experiment, mice were kept in the stereotaxic frame, spontaneous and stimulation-induced LFPs were continuously monitored, and the level of anesthesia was regularly checked. At the conclusion of all recordings, animals were deeply anesthetized, transcardially perfused, and their brains removed for histological analysis.

In each experiment, LFPs were amplified and filtered between 1 and $300 \mathrm{~Hz}$ using the Grass P55 AC Differential Amplifier (Grass Technologies) with an additional notch filter at $60 \mathrm{~Hz}$. The signal was simultaneously sampled at a rate of $1 \mathrm{kHz}$ and stored on a computer via a CED Micro1401-3 interface and Spike2 software (Cambridge Electronic Design). For quantitative offline analyses, LFPs were subjected to fast Fourier transform at a spectral resolution of $0.24 \mathrm{~Hz}$. Computing stimulation-induced hippocampal $\theta$ power was done by 
summing power values between $3 \mathrm{~Hz}$ and $9 \mathrm{~Hz}$, and peak frequency was identified by determining where the peak power occurred. To avoid possible stimulation artifacts, the first second of each 6-second-long stimulation episode was omitted in all analyses. Total $\gamma$ power during spontaneous lateral hypothalamic and hippocampal activity was computed using the same method, except the signal was band-pass filtered at between $30 \mathrm{~Hz}$ and $90 \mathrm{~Hz}$. The $\theta$ phase- $\gamma$ amplitude coupling during hippocampal stimulation was assessed using a modulation index (MI), as described previously $(62,92)$.

Statistics. Prism 7 (GraphPad Software) was used for statistical analyses. All data were initially determined to be suitable for parametric analysis according to normality and homoscedasticity. Comparisons between 2 groups were assessed with 2-tailed $t$ test, while comparisons among 3 or more groups were assessed with 1-way random effects ANOVA except where otherwise specified. The comparisons of expression of LTP between control and DIO mice were performed with 2-way ANOVA and post hoc Holm-Šidák test. Statistical differences in the relationship between elicited $\theta$ power or peak frequency and current intensity were assessed by 2-way ANOVA, while changes in spontaneous $\gamma$ power were tested with 1-way ANOVA. For all post hoc comparisons, Tukey's test was used. For statistical analyses of $\theta$ phase $-\gamma$ amplitude coupling in the hippocampus, the average $\mathrm{MI}$ in either low $\gamma(30-50 \mathrm{~Hz})$ or high $\gamma(65-95 \mathrm{~Hz})$ was calculated for each animal, grouped, and compared using a 1-way ANOVA. Data are represented as mean \pm SEM, and differences were considered significant at $P<0.05$.

Study approval. All animal procedures were performed in strict accordance with the NIH Guide for the Care and Use of Laboratory Animals (National Academies Press, 2011) and were approved by the Yale University Animal Care and Use Committee.

\section{Author contributions}

XBG conceived the original idea, and TLH provided essential input to the overall research plan. XBG, FH, and Y Tan designed the overall experimental plan. FH, Y Tan, MW, and Y Tu designed, performed and analyzed experiments for HFD treatment, the forced CWS test, and immunocytochemical experiments. Y Tan, MW, and Y Tu performed cocaine CPP with the collaboration of AML and MRP. Y Tan performed microinjection of viral vectors in Hcrt-Cre mice with the collaboration of WH and IDA. LDL provided collaboration with the generation of Hcrt-Cre mice. MS, CK, and MH designed, performed, and analyzed in vivo electrophysiological experiments. ZWL and XBG designed and performed in vitro electrophysiological experiments. LL provided input for statistical analyses of experimental results. Y Tan, $\mathrm{FH}$, and XBG wrote the manuscript, and TLH, MRP, IDA, and LDL provided helpful input for the preparation of the manuscript. The order of co-first authors appearing in the manuscript was decided by discussions among the two first authors and the corresponding author.

\section{Acknowledgments}

The authors would like to thank Hayde Sanchez and Jane Taylor (Department of Psychiatry, Yale University) for technical assistance and Marya Shanabrough for assistance with the manuscript. This study is supported by NIH grants DA040782 (to XBG); DA046160 (to XBG and TLH); DA050986, DA037566, and DA14241 (to MRP); and MH087592 (to LDL).

Address correspondence to: Xiao-Bing Gao, Department of Comparative Medicine, Yale University School of Medicine, 333 Cedar Street, New Haven, Connecticut 06520, USA. Phone: 203.785.2340; Email: xiao-bing.gao@yale.edu.
1. Johnson PM, Kenny PJ. Dopamine D2 receptors in addiction-like reward dysfunction and compulsive eating in obese rats. Nat Neurosci. 2010;13(5):635-641.

2. Brown RM, et al. Addiction-like synaptic impairments in diet-induced obesity. Biol Psychiatry. 2017;81(9):797-806.

3. Sharma S, Fulton S. Diet-induced obesity promotes depressive-like behaviour that is associated with neural adaptations in brain reward circuitry. Int J Obes (Lond). 2013;37(3):382-389.

4. Dutheil S, Ota KT, Wohleb ES, Rasmussen K, Duman RS. High-fat diet induced anxiety and anhedonia: impact on brain homeostasis and inflammation. Neuropsychopharmacology. 2016;41(7):1874-1887.

5. Vgontzas AN, Bixler EO, Tan TL, Kantner D, Martin LF, Kales A. Obesity without sleep apnea is associated with daytime sleepiness. Arch Intern Med. 1998;158(12):1333-1337.

6. Perron IJ, Pack AI, Veasey S. Diet/energy balance affect sleep and wakefulness independent of body weight. Sleep. 2015;38(12):1893-1903.

7. Zhang Z, Manson KF, Schiller D, Levy I. Impaired associative learning with food rewards in obese women. Curr Biol. 2014;24(15):1731-1736.

8. Jacka FN, Cherbuin N, Anstey KJ, Sachdev P, Butterworth P. Western diet is associated with a smaller hippocampus: a longitudinal investiga- tion. BMC Med. 2015;13:215

9. Horstmann A. It wasn't me; it was my brain - obesity-associated characteristics of brain circuits governing decision-making. Physiol Behav. 2017;176:125-133.

10. de Lecea L, et al. The hypocretins: hypothalamus-specific peptides with neuroexcitatory activity. Proc Natl Acad Sci U S A. 1998;95(1):322-327.

11. Sakurai T, et al. Orexins and orexin receptors: a family of hypothalamic neuropeptides and $G$ protein-coupled receptors that regulate feeding behavior. Cell. 1998;92(4):573-585.

12. Peyron C, et al. Neurons containing hypocretin (orexin) project to multiple neuronal systems. JNeurosci. 1998;18(23):9996-10015.

13. Gao XB, Horvath T. Function and dysfunction of hypocretin/orexin: an energetics point of view. Annu Rev Neurosci. 2014;37:101-116.

14. Gao XB, Hermes G. Neural plasticity in hypocretin neurons: the basis of hypocretinergic regulation of physiological and behavioral functions in animals. Front Syst Neurosci. 2015;9:142.

15. Cai XJ, et al. Hypothalamic orexin expression: modulation by blood glucose and feeding. Diabetes. 1999;48(11):2132-2137.

16. Cai XJ, et al. Food restriction selectively increases hypothalamic orexin-B levels in lactating rats. Regul Pept. 2001;97(2-3):163-168.

17. Kurose T, et al. Effects of restricted feeding on the activity of hypothalamic Orexin (OX)-A containing neurons and OX2 receptor mRNA level in the paraventricular nucleus of rats. Regul Pept. 2002;104(1-3):145-151.

18. Horvath TL, Gao XB. Input organization and plasticity of hypocretin neurons: possible clues to obesity's association with insomnia. Cell Metab. 2005;1(4):279-286.

19. Cai XJ, et al. Down-regulation of orexin gene expression by severe obesity in the rats: studies in Zucker fatty and zucker diabetic fatty rats and effects of rosiglitazone. Brain Res Mol Brain Res. 2000;77(1):131-137.

20. Beck B, Richy S, Dimitrov T, Stricker-Krongrad A. Opposite regulation of hypothalamic orexin and neuropeptide Y receptors and peptide expressions in obese Zucker rats. Biochem Biophys Res Commun. 2001;286(3):518-523.

21. Stricker-Krongrad A, Richy S, Beck B. Orexins/ hypocretins in the ob/ob mouse: hypothalamic gene expression, peptide content and metabolic effects. Regul Pept. 2002;104(1-3):11-20.

22. Wortley KE, Chang GQ, Davydova Z, Leibowitz SF. Peptides that regulate food intake: orexin gene expression is increased during states of hypertriglyceridemia. Am J Physiol Regul Integr Comp Physiol. 2003;284(6):R1454-R1465.

23. Chemelli RM, et al. Narcolepsy in orexin knockout mice: molecular genetics of sleep regulation. 
Cell. 1999;98(4):437-451.

24. Lin L, et al. The sleep disorder canine narcolepsy is caused by a mutation in the hypocretin (orexin) receptor 2 gene. Cell. 1999;98(3):365-376.

25. Nishino S, Ripley B, Overeem S, Lammers GJ, Mignot E. Hypocretin (orexin) deficiency in human narcolepsy. Lancet. 2000;355(9197):39-40.

26. Thannickal TC, et al. Reduced number of hypocretin neurons in human narcolepsy. Neuron. 2000;27(3):469-474.

27. Estabrooke IV, et al. Fos expression in orexin neurons varies with behavioral state. J Neurosci. 2001;21(5):1656-1662.

28. Lee MG, Hassani OK, Jones BE. Discharge of identified orexin/hypocretin neurons across the sleep-waking cycle. J Neurosci. 2005;25(28):6716-6720.

29. Mileykovskiy BY, Kiyashchenko LI, Siegel JM. Behavioral correlates of activity in identified hypocretin/orexin neurons. Neuron. 2005;46(5):787-798.

30. Zeitzer JM, Buckmaster CL, Parker KJ, Hauck CM, Lyons DM, Mignot E. Circadian and homeostatic regulation of hypocretin in a primate model: implications for the consolidation of wakefulness. J Neurosci. 2003;23(8):3555-3560.

31. Adamantidis AR, Zhang F, Aravanis AM, Deisseroth K, de Lecea L. Neural substrates of awakening probed with optogenetic control of hypocretin neurons. Nature. 2007;450(7168):420-424.

32. Han YM, et al. Leptin-promoted cilia assembly is critical for normal energy balance. J Clin Invest. 2014;124(5):2193-2197.

33. Oh EC, Vasanth S, Katsanis N. Metabolic regulation and energy homeostasis through the primary cilium. Cell Metab. 2015;21(1):21-31.

34. Siljee JE, et al. Subcellular localization of MC4R with ADCY3 at neuronal primary cilia underlies a common pathway for genetic predisposition to obesity. Nat Genet. 2018;50(2):180-185.

35. Kumamoto N, et al. A role for primary cilia in glutamatergic synaptic integration of adult-born neurons. Nat Neurosci. 2012;15(3):399-405, S1.

36. Guemez-Gamboa A, Coufal NG, Gleeson JG. Primary cilia in the developing and mature brain. Neuron. 2014;82(3):511-521.

37. Qiu L, LeBel RP, Storm DR, Chen X. Type 3 adenylyl cyclase: a key enzyme mediating the cAMP signaling in neuronal cilia. Int J Physiol Pathophysiol Pharmacol. 2016;8(3):95-108.

38. Li Y, Gao XB, Sakurai T, van den Pol AN. Hypocretin/Orexin excites hypocretin neurons via a local glutamate neuron-A potential mechanism for orchestrating the hypothalamic arousal system. Neuron. 2002;36(6):1169-1181.

39. Rao Y, et al. Regulation of synaptic efficacy in hypocretin/orexin-containing neurons by melanin concentrating hormone in the lateral hypothalamus. JNeurosci. 2008;28(37):9101-9110.

40. Kavalali ET. The mechanisms and functions of spontaneous neurotransmitter release. Nat Rev Neurosci. 2015;16(1):5-16.

41. Thanawala MS, Regehr WG. Presynaptic calcium influx controls neurotransmitter release in part by regulating the effective size of the readily releasable pool. J Neurosci. 2013;33(11):4625-4633.

42. Liu QS, Pu L, Poo MM. Repeated cocaine exposure in vivo facilitates LTP induction in midbrain dopamine neurons. Nature. 2005;437(7061):1027-1031.

43. Dietrich MO, et al. AgRP neurons regulate development of dopamine neuronal plasticity and nonfood-associated behaviors. Nat Neurosci. 2012;15(8):1108-1110

44. Rao Y, et al. Prolonged wakefulness induces experience-dependent synaptic plasticity in mouse hypocretin/orexin neurons. JClin Invest. 2007;117(12):4022-4033.

45. Carr KD. Food scarcity, neuroadaptations, and the pathogenic potential of dieting in an unnatural ecology: binge eating and drug abuse. Physiol Behav. 2011;104(1):162-167.

46. Kenny PJ. Common cellular and molecular mechanisms in obesity and drug addiction. Nat Rev Neurosci. 2011;12(11):638-651.

47. Bubser M, Fadel JR, Jackson LL, Meador-Woodruff JH, Jing D, Deutch AY. Dopaminergic regulation of orexin neurons. Eur J Neurosci. 2005;21(11):2993-3001.

48. Meyers RA, Zavala AR, Speer CM, Neisewander JL. Dorsal hippocampus inhibition disrupts acquisition and expression, but not consolidation, of cocaine conditioned place preference. Behav Neurosci. 2006;120(2):401-412.

49. Adinoff B, et al. Basal hippocampal activity and its functional connectivity predicts cocaine relapse. Biol Psychiatry. 2015;78(7):496-504.

50. McGlinchey EM, James MH, Mahler SV, Pantazis C, Aston-Jones G. Prelimbic to accumbens core pathway is recruited in a dopamine-dependent manner to drive cued reinstatement of cocaine seeking. J Neurosci. 2016;36(33):8700-8711.

51. Xia L, Nygard SK, Sobczak GG, Hourguettes NJ, Bruchas MR. Dorsal-CA1 hippocampal neuronal ensembles encode nicotine-reward contextual associations. Cell Rep. 2017;19(10):2143-2156.

52. Hitchcock LN, Lattal KM. Involvement of the dorsal hippocampus in expression and extinction of cocaine-induced conditioned place preference. Hippocampus. 2018;28(3):226-238.

53. Sjulson L, Peyrache A, Cumpelik A, Cassataro D, Buzsáki G. Cocaine place conditioning strengthens location-specific hippocampal coupling to the nucleus accumbens. Neuron. 2018;98(5):926-934.e5.

54. El Yacoubi M, et al. Behavioral, neurochemical, and electrophysiological characterization of a genetic mouse model of depression. Proc Natl Acad Sci U S A. 2003;100(10):6227-6232.

55. Fukui M, et al. Vmat2 heterozygous mutant mice display a depressive-like phenotype. J Neurosci. 2007;27(39):10520-10529.

56. Lyons DM, et al. Stress coping stimulates hippocampal neurogenesis in adult monkeys. Proc Natl Acad Sci U S A. 2010;107(33):14823-14827.

57. Montes P, Ruiz-Sánchez E, Calvillo M, Rojas P. Active coping of prenatally stressed rats in the forced swimming test: involvement of the Nurr1 gene. Stress. 2016;19(5):506-515.

58. Date Y, et al. Orexins, orexigenic hypothalamic peptides, interact with autonomic, neuroendocrine and neuroregulatory systems. Proc Natl Acad Sci U S A. 1999;96(2):748-753.

59. Trivedi P, Yu H, MacNeil DJ, Van der Ploeg LH, Guan XM. Distribution of orexin receptor mRNA in the rat brain. FEBS Lett. 1998;438(1-2):71-75.
60. Gerashchenko D, Salin-Pascual R, Shiromani PJ. Effects of hypocretin-saporin injections into the medial septum on sleep and hippocampal theta. Brain Res. 2001;913(1):106-115.

61. Marcus JN, et al. Differential expression of orexin receptors 1 and 2 in the rat brain. JComp Neurol. 2001;435(1):6-25.

62. Stoiljkovic M, et al. Hippocampal network dynamics in response to $\alpha 7 \mathrm{nACh}$ receptors activation in amyloid- $\beta$ overproducing transgenic mice. Neurobiol Aging. 2016;45:161-168.

63. MacLaren DA, et al. Clozapine N-oxide administration produces behavioral effects in Long-Evans rats: implications for designing DREADD experiments. eNeuro. 2016;3(5):ENEURO.0219-16.2016.

64. Manvich DF, et al. The DREADD agonist clozapine N-oxide (CNO) is reverse-metabolized to clozapine and produces clozapine-like interoceptive stimulus effects in rats and mice. Sci Rep. 2018;8(1):3840.

65. Di Sebastiano AR, Coolen LM. Orexin and natural reward: feeding, maternal, and male sexual behavior. Prog Brain Res. 2012;198:65-77.

66. Boutrel B, et al. Role for hypocretin in mediating stress-induced reinstatement of cocaine-seeking behavior. Proc Natl Acad Sci U S A. 2005;102(52):19168-19173.

67. Harris GC, Wimmer M, Aston-Jones G. A role for lateral hypothalamic orexin neurons in reward seeking. Nature. 2005;437(7058):556-559.

68. Yeoh JW, James MH, Jobling P, Bains JS, Graham BA, Dayas CV. Cocaine potentiates excitatory drive in the perifornical/lateral hypothalamus. JPhysiol (Lond). 2012;590(16):3677-3689.

69. Rao Y, et al. Repeated in vivo exposure of cocaine induces long-lasting synaptic plasticity in hypocretin/orexin-producing neurons in the lateral hypothalamus in mice. J Physiol (Lond). 2013;591(7):1951-1966.

70. Campioni MR, Xu M, McGehee DS. Stress-induced changes in nucleus accumbens glutamate synaptic plasticity. J Neurophysiol. 2009;101(6):3192-3198.

71. Wang Q, Wang M, Whim MD. Neuropeptide y gates a stress-induced, long-lasting plasticity in the sympathetic nervous system. J Neurosci. 2013;33(31):12705-12717.

72. Commons KG, Cholanians AB, Babb JA, Ehlinger DG. The rodent forced swim test measures stress-coping strategy, not depression-like behavior. ACS Chem Neurosci. 2017;8(5):955-960.

73. Molendijk ML, de Kloet ER. Coping with the forced swim stressor: Current state-of-the-art. Behav Brain Res. 2019;364:1-10.

74. Kovács KJ, Sawchenko PE. Sequence of stress-induced alterations in indices of synaptic and transcriptional activation in parvocellular neurosecretory neurons. J Neurosci. 1996;16(1):262-273.

75. Robison AJ, Nestler EJ. Transcriptional and epigenetic mechanisms of addiction. Nat Rev Neurosci. 2011;12(11):623-637.

76. Cristino L, et al. Obesity-driven synaptic remodeling affects endocannabinoid control of orexinergic neurons. Proc Natl Acad Sci U S A. 2013;110(24):E2229-E2238.

77. Linehan V, Fang LZ, Hirasawa M. Short-term high-fat diet primes excitatory synapses for long-term depression in orexin neurons. JPhysiol 
(Lond). 2018;596(2):305-316.

78. Jais A, Brüning JC. Hypothalamic inflammation in obesity and metabolic disease. JClin Invest. 2017;127(1):24-32.

79. Timper K, Brüning JC. Hypothalamic circuits regulating appetite and energy homeostasis: pathways to obesity. Dis Model Mech. 2017;10(6):679-689.

80. García-Cáceres C, et al. Role of astrocytes, microglia, and tanycytes in brain control of systemic metabolism. Nat Neurosci. 2019;22(1):7-14.

81. Heyward FD, et al. Obesity weighs down memory through a mechanism involving the neuroepigenetic dysregulation of Sirt1. J Neurosci. 2016;36(4):1324-1335.

82. Giardino WJ, Eban-Rothschild A, Christoffel DJ, Li SB, Malenka RC, de Lecea L. Parallel circuits from the bed nuclei of stria terminalis to the lateral hypothalamus drive opposing emotional states. Nat Neurosci. 2018;21(8):1084-1095. 83. González JA, Jensen LT, Iordanidou P, Strom M, Fugger L, Burdakov D. Inhibitory interplay between orexin neurons and eating. Curr Biol. 2016;26(18):2486-2491.

84. Han W, et al. A neural circuit for gut-induced reward. Cell. 2018;175(3):665-678.e23.

85. Parsons MP, Hirasawa M. ATP-sensitive potassium channel-mediated lactate effect on orexin neurons: implications for brain energetics during arousal. J Neurosci. 2010;30(24):8061-8070.

86. Liu ZW, Gan G, Suyama S, Gao XB. Intracellular energy status regulates activity in hypocretin/orexin neurones: a link between energy and behavioural states. J Physiol (Lond). 2011;589(17):4157-4166.

87. Paxinos G, Franklin KBJ, eds. The Mouse Brain in Stereotaxic Coordinates. Academic Press; 2001.

88. Wess J, Nakajima K, Jain S. Novel designer recep- tors to probe GPCR signaling and physiology. Trends Pharmacol Sci. 2013;34(7):385-392.

89. Alexander GM, et al. Remote control of neuronal activity in transgenic mice expressing evolved G protein-coupled receptors. Neuron. 2009;63(1):27-39.

90. Sasaki K, Suzuki M, Mieda M, Tsujino N, Roth B, Sakurai T. Pharmacogenetic modulation of orexin neurons alters sleep/wakefulness states in mice. PLoS One. 2011;6(5):e20360.

91. Purohit K, et al. Pharmacogenetic manipulation of the nucleus accumbens alters binge-like alcohol drinking in mice. Alcohol Clin Exp Res. 2018;42(5):879-888.

92. Tort AB, Komorowski RW, Manns JR, Kopell NJ, Eichenbaum H. Theta-gamma coupling increases during the learning of item-context associations. Proc Natl Acad Sci US A. 2009;106(49):20942-20947. 